\title{
Utilization Barriers and Medical Outcomes Commensurate With the Use of Telehealth Among Older Adults: Systematic Review
}

Clemens Kruse*, MSIT, MHA, MBA, PhD; Joanna Fohn", BS, MHA; Nakia Wilson*, BA, MHA; Evangelina Nunez Patlan", BS, MHA; Stephanie Zipp", BS, MHA; Michael Mileski ${ }^{*}$, DC, MHA, MPH, MSHEd

School of Health Administration, Texas State University, San Marcos, TX, United States

*all authors contributed equally

Corresponding Author:

Clemens Kruse, MSIT, MHA, MBA, PhD

School of Health Administration

Texas State University

601 University Dr

Encino Hall 250

San Marcos, TX, 78666

United States

Phone: 12103554742

Email: scottkruse@txstate.edu

\section{Abstract}

Background: Rising telehealth capabilities and improving access to older adults can aid in improving health outcomes and quality of life indicators. Telehealth is not being used ubiquitously at present.

Objective: This review aimed to identify the barriers that prevent ubiquitous use of telehealth and the ways in which telehealth improves health outcomes and quality of life indicators for older adults.

Methods: This systematic review was conducted and reported in accordance with the Kruse protocol and the Preferred Reporting Items for Systematic Reviews and Meta-Analyses (PRISMA) guidelines. Reviewers queried the following four research databases: Cumulative Index of Nursing and Allied Health Literature (CINAHL), PubMed (MEDLINE), Web of Science, and Embase (Science Direct). Reviewers analyzed 57 articles, performed a narrative analysis to identify themes, and identified barriers and reports of health outcomes and quality of life indicators found in the literature.

Results: Reviewers analyzed 57 studies across the following five interventions of telehealth: eHealth, mobile health (mHealth), telemonitoring, telecare (phone), and telehealth video calls, with a Cohen $\kappa$ of 0.75 . Reviewers identified 14 themes for barriers. The most common of which were technical literacy (25/144 occurrences, $17 \%)$, lack of desire (19/144 occurrences, 13\%), and cost (11/144 occurrences, 8\%). Reviewers identified 13 medical outcomes associated with telehealth interventions. The most common of which were decrease in psychological stress (21/118 occurrences, $18 \%)$, increase in autonomy (18/118 occurrences, $15 \%)$, and increase in cognitive ability (11/118 occurrences, $9 \%)$. Some articles did not report medical outcomes (18/57, 32\%) and some did not report barriers (19/57, 33\%).

Conclusions: The literature suggests that the elimination of barriers could increase the prevalence of telehealth use by older adults. By increasing use of telehealth, proximity to care is no longer an issue for access, and thereby care can reach populations with chronic conditions and mobility restrictions. Future research should be conducted on methods for personalizing telehealth in older adults before implementation.

Trial Registration: $\quad$ P R O S P R O $\quad$ C R D 42020182162 ; https://www.crd.york.ac.uk/prospero/display_record.php?ID=CRD42020182162.

International Registered Report Identifier (IRRID): RR2-10.2196/15490

(JMIR Med Inform 2020;8(8):e20359) doi: 10.2196/20359

\section{KEYWORDS}

telehealth; telemedicine; older adults; barriers; health outcomes 


\section{Introduction}

\section{Background}

A demographic shift has been evident globally since 2015 . Specifically, the aging population has been growing at a rapid rate and has been predicted to reach $22 \%$ by the year 2050 [1]. In fact, the World Health Organization (WHO) estimates that during 2020, adults aged 60 years or older will outnumber children aged 5 years or younger [1]. The United States Census Bureau published a graphic on March 13, 2018, depicting the population pyramid from 1960 and comparing it with the 2060 prediction [2]. The graphic demonstrated the gradual change of the US population pyramid to a pillar shape [2]. This graphic is key to understanding the demands on the health care system in the area of geriatric, long-term, and end-of-life care, because it highlights the larger number of older adults living longer lives. By 2030, 60 million people in the "baby boomer" generation (born between 1946 and 1964) will have reached 65 years of age or older and will be eligible for age-related state entitlements in most countries [3,4]. This demographic shift is an impending issue facing health care, as geriatric, long-term, and end-of-life care will experience a surge in demand. Health care organizations and their providers must find ways to effectively allocate resources and provide the right care at the right time and at the right place [5].

Telemedicine has the potential to increase access among elderly people and relieve the stress regarding care for the unusually large number of elderly people. The WHO defines telemedicine as "healing from a distance." More specifically, it is healing through the use of information and communication technologies "to improve patient outcomes by increasing access to care and medical information" [6]. The WHO also does not differentiate between the terms telemedicine and telehealth.

There has not been much work on the use of telehealth based on age; however, we know that a technology gap or digital divide exists. It is established by tiers of race, age, and economic disparities [7]. In the United States, for instance, the elder-care entitlement Medicare imposes restrictions on the use of telehealth for the primary population [8]. The Coronavirus Aid, Relieve, and Economic (CARES) Act provides a regulatory waiver to extend reimbursements to telemedicine, but this is only a relief act and not permanent legislation [9]. Previous reviews have investigated facilitators and barriers to the adoption of telehealth, the use of eHealth and mobile health (mHealth) tools in health promotion and primary prevention among older adults, and patient satisfaction with telehealth interventions [10-12]. A narrative analysis on mHealth solutions for the aging population used a generational analysis that included culture and trust of other people and a distrust of technology [13]. This work noted an increase in the use of technology for health purposes and an increase in the use of the internet for health purposes. It also noted concerns of security and privacy and technical troubleshooting. A review from 6 years ago spanned 10 years, analyzed 14 articles, and focused on older adults over 65 years old [10]. The most recent review on a topic most like this work was published 5 years ago, spanned 10 years, analyzed 45 articles, and focused on older adults aged over 50 years [11].
With an aging population, telehealth services are becoming more common to aid in independent living and health management [14]. An example of telehealth is virtual home health care, where health care providers provide guidance in specific procedures while the patients are in the comfort of their home. Telehealth programs can improve access to health care and have a positive effect on patients' medical outcomes, especially for the treatment of chronic illnesses in vulnerable populations, such as elderly people [15]. Utilizing age-friendly technology could improve the care providers give to older adults through telehealth services and improve the usability of telehealth for older adults [16]. It is essential to first understand the barriers that affect the usability of telehealth services among older adults in order to find opportunities for improving health outcomes. Barriers to using telehealth can affect the accessibility of health services to older adults. When it comes to technology, older adults are often stereotyped as laggards in technology adoption [7]. However, owing to rising telehealth capabilities, improvement of access, especially to older adults, can aid in improving health outcomes [15]. Understanding the perspectives of older adults is important when evaluating telehealth barriers because older adults generally develop different perspectives compared with those of other age demographics [16]. Other studies on this topic have focused on conditions like depression, heart failure, and falls [17-19]. However, no review has looked at medical outcomes, including indicators of quality of life, that come as a benefit of using telehealth and the barriers that exist to the use of telehealth internationally. This review intends to examine these issues and what has changed in telehealth for older adults in the last 5 years.

\section{Objectives}

The purpose of this systematic review was to evaluate the current literature to help identify and understand health-related quality of life enhancers and general health outcomes that are commensurate with and barriers to the use of telehealth services by older adults. Health outcomes, including quality of life enhancers, provide the "so what" to the use of telehealth modalities. Recognizing barriers can help develop solutions for broadening the use of telehealth services in older adults. During the COVID-19 crisis, providers and patients alike were thrust into the world of telehealth. An overview of the benefits and barriers would be helpful to those deciding whether to continue the use of telehealth modalities.

\section{Methods}

\section{Protocol and Registration}

This review used the Kruse protocol published in 2019 and the Preferred Reporting Items for Systematic Reviews and Meta-Analyses (PRISMA) guidelines [20,21]. The review was registered with PROSPERO on May 2, 2020 (ID: CRD42020182162). In accordance with the rules at PROSPERO, the registration was completed before analysis began.

\section{Eligibility Criteria}

Studies were eligible for this review if participants were older adults (older than 50 years), if the intervention was some form 
of telehealth (including mHealth, eHealth, and all forms of telehealth), if the authors reported either barriers to the use of telehealth or health outcomes, and if the article was published in a research journal in the English language in the last 5 years. Adults older than 50 years were chosen out of trial and error. When we initially wrote the methods for this study, we chose a more universal definition of older adults as those over 65 years of age. Once we started filtering articles for analysis, we noticed a large number of articles that were being eliminated, despite the high level of quality of these studies. If we had stuck with age over 65 years as our screening criteria, we would have eliminated more than half of the group of articles for analysis. As a result, we chose age over 50 years, which is supported by other reviews in this field [11]. This is a limitation we list later.

\section{Information Sources}

The following four databases were queried: Cumulative Index of Nursing and Allied Health Literature (CINAHL), PubMed (MEDLINE), Web of Science (WoS), and Embase (Science Direct). Additionally, a specific journal search was conducted in the journal of choice for publication (Journal of Medical Internet Research). Databases were filtered for the last 5 years. Database searches occurred between February 2 and 14, 2020. A period of 5 years was chosen because it has been that long since the last review was published on a similar topic. We expect to find advances in technology and advances in adoption by elderly people because younger people who use technology regularly have advanced into the observation group of over 50 years old. We hope to find fewer barriers.

\section{Search}

Reviewers carefully analyzed the MEDLINE Medical Subject Headings (MeSH) for key terms related to telehealth and elderly people. Based on the established hierarchy of indexed terms at $\mathrm{MeSH}$ and a series of experimental searches, the final search terms were "Telehealth AND 'older adults." This combination of terms yielded the maximum number of results in all four databases. Reviewers used available filters to eliminate other reviews and focus on academic or peer-reviewed journals over the last 5 years.

\section{Study Selection}

Reviewers followed the Kruse protocol, which entails a series of three consensus meetings. The results of the first consensus meeting identified the studies for analysis. After filtering the results of the four databases to meet the eligibility criteria, all reviewers screened the abstracts of the results to ensure that articles were germane to the topic, they were actually studies (not protocols), and they contained tangible results to enable analysis toward the review's objectives. The first consensus meeting discussed whether to keep articles for analysis. The reasons for rejection included opinion article (not a study), protocol (no results), concept or design paper (no results), review, no use of telehealth, and no reporting of either outcomes or barriers. A kappa statistic was calculated from the results of this meeting [20]. Before consensus meeting number two, the group leader assigned workload to ensure that each article was analyzed by at least two reviewers. Reviewers independently analyzed articles using a piloted form. Reviewers collected several standard items used for summary, such as PICOS (Participants, Intervention, Comparison [to the control group], Outcome, Study design), and analysis, such as forms of telehealth interventions, barriers to the use of telehealth by older adults, and the medical outcomes observed in older adults using telehealth solutions [20]. After making a list of observations, reviewers attempted to make sense of the observations using a narrative analysis [22].

\section{Data Collection Process}

The group leader divided analysis workload to ensure all articles were reviewed by at least two reviewers. Reviewers independently analyzed articles using a standardized Excel spreadsheet as a piloted form for data extraction.

\section{Data Items}

The piloted form collected data, including participants, intervention, study design, results compared to a control group (where applicable), medical outcomes, sample size, bias within studies, effect size, country of origin, statistics used, barriers to the use of telehealth, and quality assessment from the John Hopkins Nursing Evidence-Based Practice (JHNEBP) rating scale, as well as general observations about the article that would help in interpretation of the results [23]. These data items were independently collected and discussed in the second consensus meeting.

\section{Risk of Bias Within and Across Studies}

General observations of bias were made about each study, such as selection bias. These observations were independently collected and discussed in the second consensus meeting. The JHNEBP rating scale was used to assess the risk and quality of each study analyzed. Within the JHNEBP rating scale, level I indicates experimental studies, randomized controlled trials (RCTs), or meta analyses of RCTs; level II indicates quasiexperimental studies; level III indicates nonexperimental studies, qualitative studies, or meta-syntheses; level IV indicates opinions of nationally recognized experts based on research evidence or expert consensus panels (systematic reviews or clinical practice guidelines); and level $\mathrm{V}$ indicates opinions of individual experts based on nonresearch evidence. There are three levels of quality of evidence, which are listed as A (high quality), B (good quality), and C (low quality or major flaws). Each of these levels define the following four thresholds: research, summative reviews, organizational opinion, and expert opinion. For instance, in level A, studies have consistent results with sufficient sample size, adequate control, and definitive conclusions. In level $\mathrm{C}$, studies have little evidence with inconsistent results and insufficient sample size, and conclusions cannot be drawn. To limit the inherent bias and limitations commensurate with low-quality studies, the ratings from the JHNEBP rating scale serve as screening criteria. Articles with evidence ratings below level IV were not accepted. Quality of evidence ratings below level B were highly suspect.

\section{Summary Measures and Additional Analysis}

The review analyzed both qualitative and quantitative methods, so the summary measures sought were not consistent. The preferred summary statistic was the risk ratio, but other summary statistics were also sufficient. The summary statistics were 
independently collected and discussed in the second consensus meeting.

A narrative analysis summarized themes for barriers, interventions, and medical outcomes. They were reported in summary statistics in affinity matrices. These themes were independently collected and discussed in the third consensus meeting. After themes were identified, interactions between themes were observed using a spreadsheet.

\section{Results}

\section{Study Selection}

Figure 1 illustrates the study selection process. A kappa statistic was calculated to measure the reliability of article selection between reviewers. The $\kappa$ value was 0.75 , representing moderate agreement [24,25].

Figure 1. Study selection process.

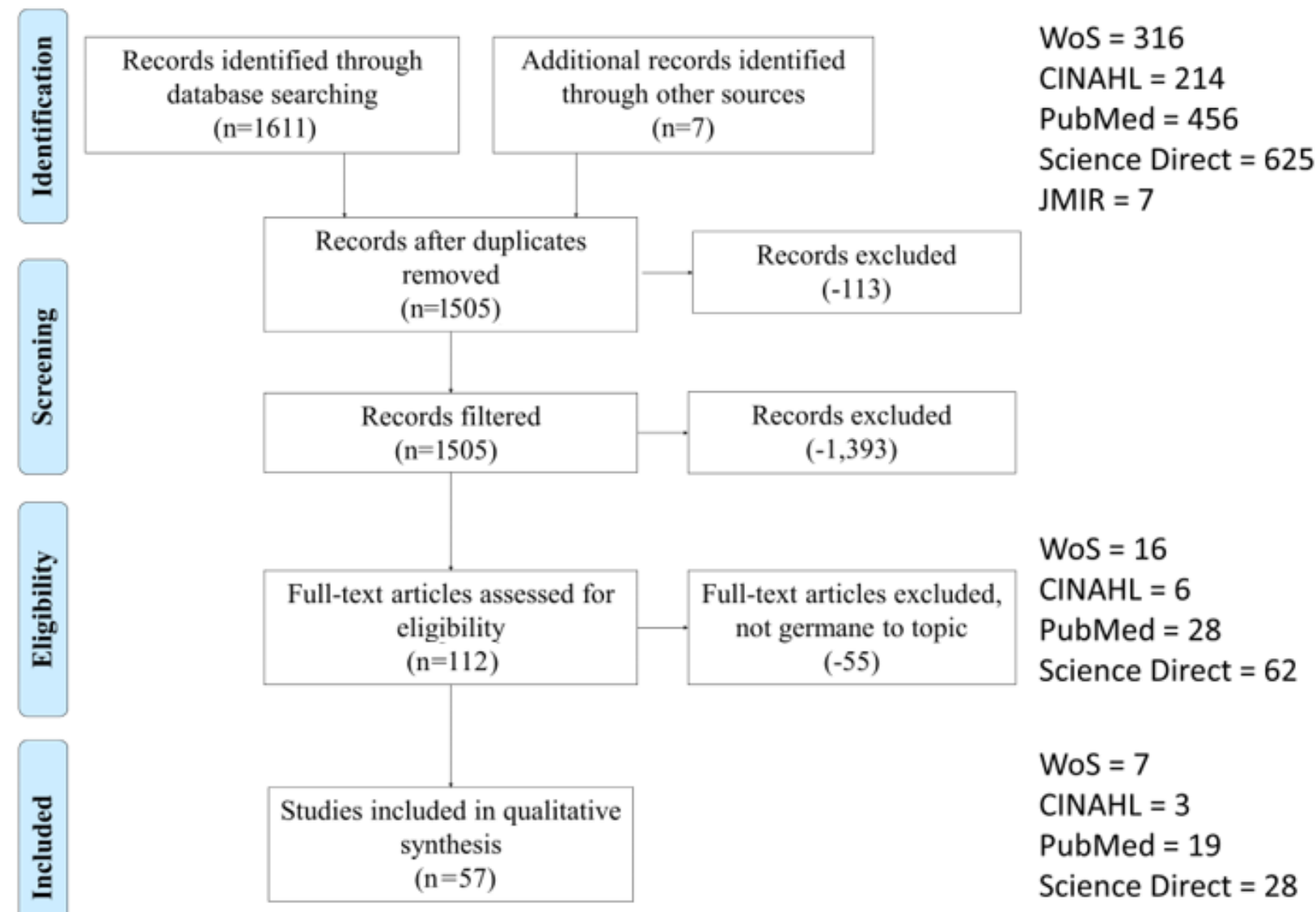

\section{Study Characteristics}

2019 [5,26-34], 2018 [4,15,16,35-46], 2017 [14,47-56], 2016

Table 1 lists the ancillary data extracted from the studies [19,57-63], and 2015 [64-76].

analyzed in reverse chronological order as follows: 2020 [26], 
Table 1. PICOS characteristics.

\begin{tabular}{|c|c|c|c|c|c|}
\hline Authors, year & Participants & Intervention & Comparator & Medical outcomes reported & Study design \\
\hline $\begin{array}{l}\text { Hamilton et al, } 2020 \\
\text { [26] }\end{array}$ & $\begin{array}{l}765 \text { older adults; } \geq 55 \\
\text { years; Medicare/Medicaid } \\
\text { beneficiaries; English } 76 \% \\
\text { (581), Spanish } 20 \% \text { (153), } \\
\text { and others } 4 \% \text { (31); low } \\
\text { income }\end{array}$ & $\begin{array}{l}\text { Telemonitoring } \\
\text { Remote patient monitoring } \\
\text { (RPM): blood-pressure cuffs, } \\
\text { pulse oximeters, and body } \\
\text { weight scales } \\
\text { Telehealth Intervention Pro- } \\
\text { grams for Seniors, RPM, exten- } \\
\text { sive social wraparound ser- } \\
\text { vices, care coordination, and } \\
\text { intergenerational socialization } \\
\text { aimed at improving health care } \\
\text { options to assist low-income } \\
\text { high health risk older adults } \\
\text { who live in subsidized congre- } \\
\text { gate housing or attend local } \\
\text { community centers for older } \\
\text { adults. } \\
\text { A survey instrument was col- } \\
\text { lected each week. }\end{array}$ & None & $\begin{array}{l}\text { Hospital visits and readmis- } \\
\text { sions }\end{array}$ & $\begin{array}{l}\text { Observational } \\
\text { study }\end{array}$ \\
\hline Theis et al, 2019 [5] & $\begin{array}{l}551 \text { older adults, } \geq 60 \\
\text { years, } 51.3 \% \text { male and } \\
48.7 \% \text { female } \\
441 \text { participants }(80 \%) \text { al- } \\
\text { ready retired, } 109(19.8 \%) \\
\text { still working }\end{array}$ & eHealth & None & $\begin{array}{l}\text { Satisfaction: } 64 \% \text { (353) of } \\
\text { older adults were satisfied } \\
\text { with the health information } \\
\text { they received, } 34 \%(187) \\
\text { were neutral, and } 2 \% \text { (11) } \\
\text { were dissatisfied }\end{array}$ & $\begin{array}{l}\text { Analytical obser- } \\
\text { vational study }\end{array}$ \\
\hline $\begin{array}{l}\text { Wildenbos et al, } 2019 \\
\text { [27] }\end{array}$ & $\begin{array}{l}13 \text { older adults, } \geq 50 \text { years, } \\
\text { primarily Dutch speaking } \\
\text { Additional inclusion crite- } \\
\text { ria for App } 2 \text { : heart failure } \\
\text { (HF) patient and chronic } \\
\text { obstructive pulmonary dis- } \\
\text { ease }\end{array}$ & $\begin{array}{l}\text { mHealth }^{\mathrm{a}} \\
\text { Investigated these interaction } \\
\text { issues in two different case } \\
\text { studies; an app for older adults } \\
\text { facilitating their hospital ap- } \\
\text { pointment attendance (App 1) } \\
\text { and a self-monitoring app for } \\
\text { chronically ill older patients }\end{array}$ & None & $\begin{array}{l}\text { Cognitive impairment was } \\
\text { reported but not compared } \\
\text { with a control. }\end{array}$ & Case study \\
\hline $\begin{array}{l}\text { Jakobsson et al, } 2019 \\
{[28]}\end{array}$ & $\begin{array}{l}9 \text { older adults, } 65-85 \text { years, } \\
\text { cognitive impairment of } \\
\text { different origin (eg, stroke, } \\
\text { dementia, and mild cogni- } \\
\text { tive impairment) }\end{array}$ & $\begin{array}{l}\text { Telehealth, smartphone, com- } \\
\text { puter, and landline }\end{array}$ & None & $\begin{array}{l}\text { Cognitive impairment was } \\
\text { reported but not compared } \\
\text { with a control. }\end{array}$ & Qualitative study \\
\hline Karlsen et al, 2019 [29] & $\begin{array}{l}18 \text { older adults, } \geq 60 \text { years, } \\
\text { living in their own homes } \\
\text { and having recently re- } \\
\text { ceived telecare service } \\
\text { (within the last } 0-3 \\
\text { months), received home } \\
\text { care services, Norwegian } \\
\text { speaking, no limitations } \\
\text { considering disease or } \\
\text { chronic conditions }\end{array}$ & $\begin{array}{l}\text { Telemonitoring } \\
\text { Personal alarm (16), light sen- } \\
\text { sors (3), stove alarm (4), GPS } \\
\text { tracking (3), medication re- } \\
\text { minders (8), bed sensors (1), } \\
\text { door sensor (2), video surveil- } \\
\text { lance (2) }\end{array}$ & None & $\begin{array}{l}\text { Safety, satisfaction, securi- } \\
\text { ty, independence, responsi- } \\
\text { bility, mindfulness of failty }\end{array}$ & Qualitative study \\
\hline
\end{tabular}




\begin{tabular}{|c|c|c|c|c|c|}
\hline Authors, year & Participants & Intervention & Comparator & Medical outcomes reported & Study design \\
\hline Coley et al, 2019 [30] & $\begin{array}{l}341 \text { (quantitative) and } 46 \\
\text { (qualitative) older adults; } \\
\geq 65 \text { years; Finland, France, } \\
\text { and Netherlands; response } \\
\text { rate } 79 \% \text { (Finland: } 81 \%, \\
\text { France: } 72 \% \text {, Netherlands: } \\
87 \%, P=.04 \text { ); } 48 \%(164) \\
\text { male; } 51 \% \text { (174) universi- } \\
\text { ty-level education }\end{array}$ & $\begin{array}{l}\text { eHealth } \\
\text { Participants were randomized } \\
\text { to either an interactive internet } \\
\text { platform designed to encourage } \\
\text { goal setting and lifestyle } \\
\text { changes with the remote sup- } \\
\text { port of a lifestyle coach or a } \\
\text { control platform with basic } \\
\text { health information but no inter- } \\
\text { active features or coach sup- } \\
\text { port. Owing to the nature of the } \\
\text { intervention, complete double } \\
\text { blinding was not possible, but } \\
\text { masking was attempted by in- } \\
\text { forming participants that they } \\
\text { would be randomized to one of } \\
\text { two internet platforms (without } \\
\text { further details on the content). }\end{array}$ & Control & Not reported & $\begin{array}{l}\text { Cross-sectional } \\
\text { mixed-methods } \\
\text { randomized con- } \\
\text { trolled trial } \\
(\mathrm{RCT})\end{array}$ \\
\hline $\begin{array}{l}\text { Giesbrecht \& Miller, } \\
2019 \text { [31] }\end{array}$ & $\begin{array}{l}18 \text { older adults, } \geq 50 \text { years, } \\
\text { resided in the community, } \\
\text { self-propelled using both } \\
\text { hands at least } 1 \text { hour per } \\
\text { day inside and outside } \\
\text { their home, English speak- } \\
\text { ing }\end{array}$ & $\begin{array}{l}\text { eHealth } \\
\text { The treatment group incorporat- } \\
\text { ed two in-person training ses- } \\
\text { sions with a trainer and } 4 \text { weeks } \\
\text { of monitored home training us- } \\
\text { ing a computer tablet (mHealth) } \\
\text { wheelchair skills program. The } \\
\text { control group did not receive } \\
\text { skills training, as is typical } \\
\text { practice with this population. }\end{array}$ & Control & Skill capacity and safety & RCT \\
\hline $\begin{array}{l}\text { Brodbeck et al, } 2019 \\
\text { [32] }\end{array}$ & $\begin{array}{l}110 \text { older adults, }>50 \\
\text { years, } 79 \% \text { ( } 87) \text { female }\end{array}$ & $\begin{array}{l}\text { eHealth } \\
\text { Internet-based self-help inter- } \\
\text { vention for prolonged grief } \\
\text { symptoms after spousal bereave- } \\
\text { ment or separation/divorce }\end{array}$ & Control & $\begin{array}{l}\text { Grief, depression, psycho- } \\
\text { logical distress, embitter- } \\
\text { ment, loneliness, and life } \\
\text { satisfaction }\end{array}$ & $\begin{array}{l}\text { Mixed methods, } \\
\text { RCT }\end{array}$ \\
\hline Mosley et al, 2019 [33] & $\begin{array}{l}112 \text { older adults, } \geq 60 \\
\text { years, } 58 \%(65) \text { female, } \\
\text { English speaking }\end{array}$ & $\begin{array}{l}\text { eHealth } \\
\text { Etymotic home hearing test } \\
\text { compared with traditional } \\
\text { manual audiometry }\end{array}$ & Control & Not reported & $\begin{array}{l}\text { Quasiexperimen- } \\
\text { tal study }\end{array}$ \\
\hline Jensen et al, 2019 [34] & $\begin{array}{l}20 \text { older adults, hip frac- } \\
\text { ture }\end{array}$ & $\begin{array}{l}\text { eHealth } \\
\text { "My Hip Fracture Journey" on } \\
\text { iPad (provided) education } \\
\text { through pictographs, video } \\
\text { clips, illustrated exercises, and } \\
\text { written information. This was } \\
\text { used to augment home visits } \\
\text { and subsequent interviews. }\end{array}$ & None & Autonomy and self-care & Qualitative study \\
\hline Rasche et al, 2018 [15] & $\begin{array}{l}576 \text { older adults, } \geq 60 \\
\text { years, } 48.7 \%(280) \text { female, } \\
\text { German speaking }\end{array}$ & $\begin{array}{l}\text { eHealth } \\
\text { The national survey queried the } \\
\text { use of health apps and their } \\
\text { perceived usefulness. }\end{array}$ & Control & Not reported & $\begin{array}{l}\text { Quasiexperimen- } \\
\text { tal study }\end{array}$ \\
\hline Portz et al, 2018 [35] & $\begin{array}{l}30 \text { older adults, } \geq 60 \text { years, } \\
\text { location at the University } \\
\text { of Colorado Hospital and } \\
\text { the University Hospital } \\
\text { Cleveland Medical Center } \\
\text { in Cleveland (Ohio), } 60 \% \\
\text { (18) female, } 63 \% \text { (19) } \\
\text { black people }\end{array}$ & $\begin{array}{l}\text { mHealth } \\
\text { The HF app was developed to } \\
\text { allow patients to track their } \\
\text { symptoms of HF. Thirty older } \\
\text { adults completed an acceptabil- } \\
\text { ity survey after using the mo- } \\
\text { bile app. The survey used Lik- } \\
\text { ert items and open-ended feed- } \\
\text { back questions. }\end{array}$ & None & $\begin{array}{l}\text { Awareness of the condition } \\
\text { and self-care }\end{array}$ & $\begin{array}{l}\text { Quantitative ac- } \\
\text { ceptability survey } \\
\text { analysis }\end{array}$ \\
\hline
\end{tabular}




\begin{tabular}{|c|c|c|c|c|c|}
\hline Authors, year & Participants & Intervention & Comparator & Medical outcomes reported & Study design \\
\hline Castro et al, 2018 [36] & $\begin{array}{l}501 \text { older adults, } \geq 65 \\
\text { years, Medicare population }\end{array}$ & $\begin{array}{l}\text { eHealth } \\
\text { Participants were matched into } \\
\text { geographically based small } \\
\text { groups with an assigned health } \\
\text { coach, and they began the pro- } \\
\text { gram at the same time. Group } \\
\text { members were connected to } \\
\text { each other through a private } \\
\text { online social forum where they } \\
\text { could post comments and } \\
\text { questions, engage in health } \\
\text { coach-moderated discussions, } \\
\text { and provide social support to } \\
\text { one another. } \\
\text { Using internet-enabled devices } \\
\text { (laptop, tablet, or smartphone), } \\
\text { program participants were able } \\
\text { to asynchronously complete } \\
\text { weekly interactive curriculum } \\
\text { lessons, reflections, and goal- } \\
\text { setting activities in relation to } \\
\text { the weekly topic. }\end{array}$ & Pretest & $\begin{array}{l}\text { Weight: participants lost } \\
\text { an average of } 13-14 \\
\text { pounds }(8 \%) \\
\mathrm{HbA}_{1 \mathrm{c}}: 0.14 \% \text { absolute } \\
\text { decrease at } 6 \text { months and } \\
12 \text { months }(P<.001) \\
\text { Cholesterol: mean reduc- } \\
\text { tion of }-12.92 \mathrm{mg} / \mathrm{dL} \\
(P<.001) \text {. }\end{array}$ & $\begin{array}{l}\text { Single-arm } \\
\text { pretest/posttest } \\
\text { design }\end{array}$ \\
\hline Joe et al, 2018 [37] & $\begin{array}{l}43 \text { older adults, } 70 \%(30) \\
\text { female }\end{array}$ & $\begin{array}{l}\text { eHealth } \\
\text { A focus-group method was } \\
\text { used to brainstorm designs for } \\
\text { telehealth for older adults. }\end{array}$ & None & Not reported & $\begin{array}{l}\text { Qualitative analy- } \\
\text { sis study (focus } \\
\text { groups) }\end{array}$ \\
\hline Dham et al, 2018 [4] & $\begin{array}{l}134 \text { older adults, } 60 \%(80) \\
\text { female }\end{array}$ & $\begin{array}{l}\text { Telemedicine } \\
\text { Telepsychiatry assessments }\end{array}$ & None & Not reported & $\begin{array}{l}\text { Mixed-methods } \\
\text { cross-sectional } \\
\text { cohort study with } \\
\text { retrospective } \\
\text { chart review and } \\
\text { prospective feed- } \\
\text { back survey }\end{array}$ \\
\hline Paige et al, 2018 [16] & $\begin{array}{l}384 \text { older adults, } 74.3 \% \\
(285) \text { female, } 57.7 \%(222) \\
\text { Caucasian people, } 42.3 \% \\
\text { (162) black people }\end{array}$ & $\begin{array}{l}\text { eHealth } \\
\text { eHealth awareness and eHealth } \\
\text { literacy scale }\end{array}$ & None & Not reported & $\begin{array}{l}\text { Qualitative mea- } \\
\text { surement invari- } \\
\text { ance study }\end{array}$ \\
\hline Cajita et al, 2018 [38] & $\begin{array}{l}10 \text { older adults, } \geq 65 \text { years, } \\
\text { history of HF, spoke En- } \\
\text { glish, difficulty with mo- } \\
\text { bile technology }\end{array}$ & mHealth & None & Not reported & $\begin{array}{l}\text { Descriptive ex- } \\
\text { ploratory study }\end{array}$ \\
\hline Harte et al, 2018 [39] & $\begin{array}{l}22 \text { older adults, }>65 \text { years, } \\
\text { difficulty using smart- } \\
\text { phones }\end{array}$ & $\begin{array}{l}\text { mHealth } \\
\text { Training on a smartphone- } \\
\text { based fall detection and preven- } \\
\text { tion system }\end{array}$ & None & Not reported & $\begin{array}{l}\text { Usability and } \\
\text { learnability case } \\
\text { study }\end{array}$ \\
\hline $\begin{array}{l}\text { Gordon \& Hornbrook, } \\
2018 \text { [40] }\end{array}$ & $\begin{array}{l}2602 \text { older adults, }>65 \\
\text { years, } 54 \%(1,405) \text { female, } \\
79 \%(2,056) \text { Caucasian } \\
\text { people }\end{array}$ & $\begin{array}{l}\text { eHealth } \\
\text { Online forms, online tracking } \\
\text { systems, and patient portal }\end{array}$ & None & Not reported & $\begin{array}{l}\text { Mixed-methods } \\
\text { cross-sectional } \\
\text { study }\end{array}$ \\
\hline Bao et al, 2018 [41] & $\begin{array}{l}12 \text { older adults, } \geq 65 \text { years, } \\
75 \% \text { (8) female }\end{array}$ & $\begin{array}{l}\text { eHealth } \\
\text { Online training }\end{array}$ & Pretest & $\begin{array}{l}\text { Sensory organization test, } \\
\text { mini balance evaluation } \\
\text { system test, five times sit } \\
\text { to stand test, and no statis- } \\
\text { tical significance in other } \\
\text { clinical outcomes }\end{array}$ & $\begin{array}{l}\text { Pretest posttest } \\
\text { true experiment }\end{array}$ \\
\hline Egede et al, 2018 [42] & $\begin{array}{l}241 \text { older adults, }>63 \\
\text { years, } 98 \%(236) \text { male, } \\
60 \% \text { (144) Caucasian peo- } \\
\text { ple, veterans having major } \\
\text { depressive disorder }\end{array}$ & $\begin{array}{l}\text { Telemedicine } \\
\text { Telepsychotherapy }\end{array}$ & Control & $\begin{array}{l}\text { Baseline depression sever- } \\
\text { ity, generalized anxiety } \\
\text { disorder, alcohol misuse, } \\
\text { cannabis misuse, and } \\
\text { cannabis dependence }\end{array}$ & RCT \\
\hline
\end{tabular}




\begin{tabular}{|c|c|c|c|c|c|}
\hline Authors, year & Participants & Intervention & Comparator & Medical outcomes reported & Study design \\
\hline $\begin{array}{l}\text { Platts-Mills et al, } 2018 \\
\text { [43] }\end{array}$ & $\begin{array}{l}75 \text { older adults, }<50 \text { years, } \\
\text { musculoskeletal pain }\end{array}$ & $\begin{array}{l}\text { Telecare } \\
\text { Telephone call and protocol- } \\
\text { guided follow up }\end{array}$ & Control & Pain & $\begin{array}{l}\text { Randomized con- } \\
\text { trolled pilot study }\end{array}$ \\
\hline $\begin{array}{l}\text { Lopez-Villegas et al, } \\
2018 \text { [44] }\end{array}$ & $\begin{array}{l}50 \text { older adults, }>65 \text { years, } \\
48 \%(24) \text { women, seen in } \\
\text { the cardiology clinic, using } \\
\text { a pacemaker }\end{array}$ & $\begin{array}{l}\text { Telemonitoring } \\
\text { Pacemakers }\end{array}$ & Control & $\begin{array}{l}\text { EQ-5D VAS }{ }^{b} \text { (health-relat- } \\
\text { ed quality of life) }\end{array}$ & $\mathrm{RCT}$ \\
\hline Dugas et al, 2018 [45] & 27 older adults, $>60$ years & $\begin{array}{l}\text { mHealth } \\
\text { DiaSocial for glucose control, } \\
\text { exercise, nutrition, and medica- } \\
\text { tion adherence }\end{array}$ & None & $\begin{array}{l}\text { Glucose management and } \\
\mathrm{HbA}_{1 \mathrm{c}}\end{array}$ & Pilot study \\
\hline Nalder et al, 2018 [46] & $\begin{array}{l}8 \text { older adults, }>55 \text { years, } \\
\text { type } 2 \text { diabetes }\end{array}$ & $\begin{array}{l}\text { eHealth } \\
\text { Three internet-based platforms: } \\
\text { 1. Chronic disease management } \\
\text { 2. Real-world strategy training } \\
\text { 3. Learning the ropes }\end{array}$ & None & $\begin{array}{l}\mathrm{HbA}_{1 \mathrm{c}}, \text { independence, } \\
\text { emotional support, and } \\
\text { motivation to self-manage }\end{array}$ & $\begin{array}{l}\text { Qualitative pilot } \\
\text { program }\end{array}$ \\
\hline Buck et al, 2017 [47] & $\begin{array}{l}12 \text { older adults, }>60 \text { years, } \\
42 \% \text { (5) female }\end{array}$ & $\begin{array}{l}\text { eHealth } \\
\text { PSHA, a web-based tablet-de- } \\
\text { livered intervention developed } \\
\text { internally, which encourages } \\
\text { the participant to record daily } \\
\text { medication intake, weight, and } \\
\text { time spent with a brief exercise } \\
\text { program using an aerobic step- } \\
\text { per. The tablet records daily } \\
\text { information, and the participant } \\
\text { watches a short heart health } \\
\text { educational video. }\end{array}$ & None & $\begin{array}{l}\text { Documentation for nutri- } \\
\text { tion and eating and instruc- } \\
\text { tional video exposure }\end{array}$ & $\begin{array}{l}\text { Proof-of-concept } \\
\text { trial } \\
\text { Qualitative } \\
\text { semistructured } \\
\text { interviews after } \\
\text { the study proto- } \\
\text { col }\end{array}$ \\
\hline Ware et al, 2017 [14] & $\begin{array}{l}15 \text { older adults, } \geq 50 \text { years, } \\
73 \% \text { (11) female }\end{array}$ & eHealth & None & Not reported & $\begin{array}{l}\text { Two focus } \\
\text { groups and prag- } \\
\text { matic thematic } \\
\text { analysis }\end{array}$ \\
\hline Chang et al, 2017 [48] & $\begin{array}{l}18 \text { older adults, }>65 \text { years, } \\
\text { diabetes }\end{array}$ & $\begin{array}{l}\text { Telehealth } \\
\text { Diabetes management }\end{array}$ & None & $\begin{array}{l}\text { Self-management and inde- } \\
\text { pendence }\end{array}$ & $\begin{array}{l}\text { Qualitative re- } \\
\text { search design and } \\
1-1 \text { semistruc- } \\
\text { tured interviews }\end{array}$ \\
\hline Cajita et al, 2017 [49] & $\begin{array}{l}129 \text { older adults, }>65 \\
\text { years, } 73.6 \%(95) \text { male, } \\
56.6 \%(73) \text { Caucasian } \\
\text { people }\end{array}$ & $\begin{array}{l}\text { mHealth } \\
\text { Simple linear regression was } \\
\text { used to test the relationship be- } \\
\text { tween the main study variables } \\
\text { (eHealth literacy, social influ- } \\
\text { ence, perceived financial cost, } \\
\text { perceived ease of use, and per- } \\
\text { ceived usefulness) and intention } \\
\text { to use mHealth. }\end{array}$ & None & Not reported & $\begin{array}{l}\text { Cross-sectional } \\
\text { correlational } \\
\text { study }\end{array}$ \\
\hline $\begin{array}{l}\text { LaMonica et al, } 2017 \\
\text { [50] }\end{array}$ & $\begin{array}{l}221 \text { older adults, } \geq 50 \\
\text { years, } 57.7 \%(128) \text { female }\end{array}$ & $\begin{array}{l}\text { eHealth } \\
\text { Memory aids and mental acuity } \\
\text { exercises }\end{array}$ & None & Memory & Qualitative study \\
\hline $\begin{array}{l}\text { Bahar-Fuchs et al, } 2017 \\
\text { [51] }\end{array}$ & $\begin{array}{l}45 \text { older adults; }>65 \text { years; } \\
\text { mild cognitive impairment } \\
(n=9), \text { mood-related neu- } \\
\text { ropsychiatric symptoms } \\
(n=11), \text { or both }(n=25)\end{array}$ & $\begin{array}{l}\text { eHealth } \\
\text { Tailored and adaptive computer } \\
\text { cognitive training in older } \\
\text { adults at risk for dementia }\end{array}$ & Control & $\begin{array}{l}\text { Memory, global cognition, } \\
\text { learning, and mood }\end{array}$ & RCT \\
\hline Nahm et al, 2017 [52] & $\begin{array}{l}866 \text { older adults, }>50 \\
\text { years, bone health issues }\end{array}$ & $\begin{array}{l}\text { eHealth } \\
\text { Bone Power program }\end{array}$ & Control & $\begin{array}{l}\text { Osteoporosis knowledge, } \\
\text { self-efficacy/outcome ex- } \\
\text { pectations, and exercise } \\
\text { behaviors }\end{array}$ & Two-arm RCT \\
\hline
\end{tabular}




\begin{tabular}{|c|c|c|c|c|c|}
\hline Authors, year & Participants & Intervention & Comparator & Medical outcomes reported & Study design \\
\hline $\begin{array}{l}\text { Knaevelsrud et al, } 2017 \\
\text { [53] }\end{array}$ & $\begin{array}{l}47 \text { older adults, }>50 \text { years, } \\
64.9 \%(31) \text { female, post- } \\
\text { traumatic stress disorder } \\
\text { symptoms, German speak- } \\
\text { ing }\end{array}$ & $\begin{array}{l}\text { eHealth } \\
\text { Internet-based therapist-guided } \\
\text { intervention }\end{array}$ & Control & $\begin{array}{l}\text { Comfort (from not being } \\
\text { able to see the therapist), } \\
\text { satisfaction, motivation, } \\
\text { feeling of being under- } \\
\text { stood }\end{array}$ & RCT \\
\hline $\begin{array}{l}\text { Reijnders et al, } 2017 \\
\text { [54] }\end{array}$ & $\begin{array}{l}376 \text { older adults, }>50 \\
\text { years, } 67.5 \% \text { female }\end{array}$ & $\begin{array}{l}\text { eHealth } \\
\text { Cognitive functioning }\end{array}$ & Control & $\begin{array}{l}\text { Feelings of stability, mem- } \\
\text { ory functioning, and locus } \\
\text { of control }\end{array}$ & $\mathrm{RCT}$ \\
\hline $\begin{array}{l}\text { Hamblin et al, } 2017 \\
\text { [56] }\end{array}$ & 60 older adults, $>85$ years & Telemonitoring & None & $\begin{array}{l}\text { Autonomy, awareness of } \\
\text { danger areas like gardens } \\
\text { or staircases, and safety }\end{array}$ & $\begin{array}{l}\text { Mixed-methods } \\
\text { study }\end{array}$ \\
\hline $\begin{array}{l}\text { Mageroski et al, } 2016 \\
{[55]}\end{array}$ & 25 older adults, $>50$ years & $\begin{array}{l}\text { Telemonitoring } \\
\text { Remote sensors in homes of } \\
\text { older adults }\end{array}$ & None & Not reported & $\begin{array}{l}\text { Mixed-methods } \\
\text { study }\end{array}$ \\
\hline Wang et al, 2016 [57] & $\begin{array}{l}29 \text { older adults, }>65 \text { years, } \\
71 \% \text { (21) female }\end{array}$ & $\begin{array}{l}\text { Telemonitoring } \\
\text { Wearables, mobile devices, } \\
\text { trackers, and in-home telemon- } \\
\text { itoring }\end{array}$ & None & Not reported & $\begin{array}{l}\text { Cross-sectional } \\
\text { study }\end{array}$ \\
\hline $\begin{array}{l}\text { Gordon \& Hornbrook, } \\
2016 \text { [58] }\end{array}$ & $\begin{array}{l}231,082 \text { older adults for } \\
\text { database arm, } 2602 \text { older } \\
\text { adults for survey arm }\end{array}$ & eHealth & None & Not reported & $\begin{array}{l}\text { Mixed methods, } \\
\text { database, and sur- } \\
\text { vey study }\end{array}$ \\
\hline $\begin{array}{l}\text { Williams et al, } 2016 \\
\text { [59] }\end{array}$ & $\begin{array}{l}7 \text { older adults, }>60 \text { years, } \\
\text { dementia }\end{array}$ & eHealth & None & Not reported & Pilot study \\
\hline Evans et al, 2016 [19] & $\begin{array}{l}41 \text { older adults, }>55 \text { years, } \\
57.1 \%(23) \text { female, En- } \\
\text { glish speaking }\end{array}$ & $\begin{array}{l}\text { mHealth } \\
\text { Remote monitoring, wrist } \\
\text { wearable, and wireless tablet }\end{array}$ & Control & $\begin{array}{l}\text { Documentation for weight } \\
\text { and blood pressure }\end{array}$ & $\begin{array}{l}\text { Single-arm quasi- } \\
\text { experimental } \\
\text { study }\end{array}$ \\
\hline Muller et al, 2016 [60] & $\begin{array}{l}43 \text { older adults, } \geq 55 \text { years, } \\
\text { mobile phone use, no regu- } \\
\text { lar exercise }\end{array}$ & $\begin{array}{l}\text { mHealth } \\
\text { SMS and Physical Activity for } \\
\text { Health Study }\end{array}$ & Control & $\begin{array}{l}\text { Exercise, mood, fitness, } \\
\text { health, mindfulness of the } \\
\text { importance of exercise, } \\
\text { and guilt }\end{array}$ & $\begin{array}{l}\text { Two-arm parallel } \\
\text { RCT }\end{array}$ \\
\hline Quinn et al, 2016 [61] & $\begin{array}{l}118 \text { older adults, }>50 \\
\text { years, } 66 \%(78) \text { female, } \\
\text { diabetes }\end{array}$ & $\begin{array}{l}\text { mHealth } \\
\text { Mobile diabetes intervention } \\
\text { study }\end{array}$ & Control & $\mathrm{HbA}_{1 \mathrm{c}}$ & True experiment \\
\hline $\begin{array}{l}\text { Royackers et al, } 2016 \\
{[62]}\end{array}$ & $\begin{array}{l}8 \text { older adults, caring for } \\
\text { loved ones in their last } \\
\text { days }\end{array}$ & $\begin{array}{l}\text { eHealth } \\
\text { Point of care technology } \\
\text { through eShift (home-based } \\
\text { palliative care) }\end{array}$ & None & $\begin{array}{l}\text { Comfort, independence, } \\
\text { and autonomy }\end{array}$ & $\begin{array}{l}\text { Qualitative pilot } \\
\text { study }\end{array}$ \\
\hline Duh et al, 2016 [63] & 45 older adults, $>60$ years & $\begin{array}{l}\text { Telecare } \\
\text { CareMe }\end{array}$ & None & Not reported & $\begin{array}{l}\text { Qualitative partic- } \\
\text { ipatory design }\end{array}$ \\
\hline $\begin{array}{l}\text { Depatie \& Bigbee, } 2015 \\
{[64]}\end{array}$ & $\begin{array}{l}30 \text { older adults, } \geq 60 \text { years, } \\
80 \% \text { ( } 24) \text { female }\end{array}$ & $\begin{array}{l}\text { mHealth } \\
\text { Mobile health technology for } \\
\text { older adults in rural communi- } \\
\text { ties }\end{array}$ & None & Not reported & $\begin{array}{l}\text { Mixed-methods } \\
\text { study }\end{array}$ \\
\hline Moore et al, 2015 [65] & $\begin{array}{l}26 \text { older adults, }>55 \text { years, } \\
77 \% \text { (20) male }\end{array}$ & $\begin{array}{l}\text { eHealth } \\
\text { Internet-based hearing health } \\
\text { care for older adults }\end{array}$ & None & Not reported & Training study \\
\hline Currie et al, 2015 [66] & $\begin{array}{l}168 \text { older adults, } \geq 60 \\
\text { years, living in rural areas, } \\
\text { long-term chronic pain }\end{array}$ & eHealth & None & Pain & $\begin{array}{l}\text { Mixed-methods } \\
\text { study }\end{array}$ \\
\hline
\end{tabular}




\begin{tabular}{|c|c|c|c|c|c|}
\hline Authors, year & Participants & Intervention & Comparator & Medical outcomes reported & Study design \\
\hline Grant et al, 2015 [67] & $\begin{array}{l}762 \text { older adults, }>60 \\
\text { years, } 67 \%(511) \text { female, } \\
90 \%(686) \text { Caucasian peo- } \\
\text { ple }\end{array}$ & $\begin{array}{l}\text { Telemonitoring } \\
\text { LivingWell@Home, sensors } \\
\text { (motion, bed, and humidity), } \\
\text { emergency response systems, } \\
\text { and biometric monitors (heart } \\
\text { rate, blood pressure, weight, } \\
\text { pulse oximetry, and blood glu- } \\
\text { cose) }\end{array}$ & Control & $\begin{array}{l}\text { Satisfaction, autonomy, } \\
\text { and independence }\end{array}$ & RCT \\
\hline Brenes et al, 2015 [68] & $\begin{array}{l}141 \text { older adults, } \geq 60 \\
\text { years, } 81 \% \text { (114) female, } \\
\text { living in rural areas, diag- } \\
\text { nosis of generalized anxi- } \\
\text { ety disorder (GAD) }\end{array}$ & $\begin{array}{l}\text { Telecare } \\
\text { Telephone-delivered cognitive } \\
\text { behavior therapy and tele- } \\
\text { phone-delivered nondirective } \\
\text { supportive therapy }\end{array}$ & Control & $\begin{array}{l}\text { Worry, GAD, depression, } \\
\text { and anxiety }\end{array}$ & $\mathrm{RCT}$ \\
\hline Corbett et al, 2015 [69] & $\begin{array}{l}2192 \text { older adults, } \geq 60 \\
\text { years }\end{array}$ & $\begin{array}{l}\text { eHealth } \\
\text { Online cognitive training pack- } \\
\text { age }\end{array}$ & Control & $\begin{array}{l}\text { Reasoning, verbal learn- } \\
\text { ing, and instrumental activ- } \\
\text { ities of daily living }\end{array}$ & $\mathrm{RCT}$ \\
\hline $\begin{array}{l}\text { Mavandadi et al, } 2015 \\
{[70]}\end{array}$ & $\begin{array}{l}1018 \text { older adults, } \geq 65 \\
\text { years, } 83.2 \% \text { ( } 847) \text { female, } \\
\text { community-dwelling, low- } \\
\text { income, mental health } \\
\text { symptoms }\end{array}$ & $\begin{array}{l}\text { Telecare } \\
\text { SUSTAIN care management } \\
\text { system (assessment, monitor- } \\
\text { ing, care management, and } \\
\text { brief therapies) }\end{array}$ & Control & $\begin{array}{l}\text { Depressive symptoms, } \\
\text { anxiety symptoms, and } \\
\text { MH functioning }\end{array}$ & RCT \\
\hline Egede et al, 2015 [71] & $\begin{array}{l}90 \text { older adults, } \geq 58 \text { years, } \\
98 \% \text { ( } 88) \text { male, diagnosis } \\
\text { of diabetes }\end{array}$ & $\begin{array}{l}\text { Telemedicine } \\
\text { Telepsychotherapy }\end{array}$ & Control & $\begin{array}{l}\text { Geriatric depression scale, } \\
\text { Beck depression inventory, } \\
\text { and Diagnostic and Statis- } \\
\text { tical Manual, version } 4 \\
\text { symptoms }\end{array}$ & RCT \\
\hline Chang et al, 2015 [72] & $\begin{array}{l}192 \text { older adults, }>60 \\
\text { years, } 81 \% \text { (156) female, } \\
\text { cardiology diagnoses }\end{array}$ & $\begin{array}{l}\text { Telemonitoring } \\
\text { Remote cardiology manage- } \\
\text { ment }\end{array}$ & None & $\begin{array}{l}\text { Cardiac arrhythmias detect- } \\
\text { ed and paroxysmal atrial } \\
\text { fibrillation detected }\end{array}$ & Pilot study \\
\hline Boulos et al, 2015 [73] & $\begin{array}{l}27 \text { older adults, } 31 \text { care- } \\
\text { givers, } 43 \text { healthcare pro- } \\
\text { fessionals }\end{array}$ & $\begin{array}{l}\text { eHealth } \\
\text { LiveWell Parkinson interven- } \\
\text { tion and learning modules }\end{array}$ & None & $\begin{array}{l}\text { Communication of the } \\
\text { condition with the provider }\end{array}$ & Pilot study \\
\hline $\begin{array}{l}\text { Dino \& deGuzman, } \\
2015 \text { [74] }\end{array}$ & $\begin{array}{l}82 \text { older adults, demograph- } \\
\text { ics not reported }\end{array}$ & $\begin{array}{l}\text { Telemedicine, mHealth, and } \\
\text { eHealth }\end{array}$ & None & Not reported & $\begin{array}{l}\text { Structured equa- } \\
\text { tion modeling }\end{array}$ \\
\hline Czaja et al, 2015 [75] & $\begin{array}{l}24 \text { older adults, }>60 \text { years, } \\
71 \% \text { (17) female, } 94 \%(23) \\
\text { Hispanic people, diagnosis } \\
\text { of hypertension }\end{array}$ & $\begin{array}{l}\text { Telemonitoring } \\
\text { Telehealth system that monitors } \\
\text { blood pressure and body weight }\end{array}$ & Control & $\begin{array}{l}\text { Self-management, health, } \\
\text { and independence }\end{array}$ & $\begin{array}{l}\text { Randomized feasi- } \\
\text { bility study }\end{array}$ \\
\hline Choi et al, 2015 [76] & $\begin{array}{l}42 \text { older adults, } \geq 60 \text { years, } \\
81 \% \text { (34) female, low-in- } \\
\text { come, homebound, score } \\
\text { of } 15 \text { or above on the } 24- \\
\text { item Hamilton Rating } \\
\text { Scale for Depression }\end{array}$ & $\begin{array}{l}\text { Telecare } \\
\text { Video tele-problem-solving } \\
\text { therapy (PST) to in-person PST } \\
\text { and telephone care calls }\end{array}$ & None & $\begin{array}{l}\text { Depressive symptoms, un- } \\
\text { derstanding of depression, } \\
\text { and social interaction }\end{array}$ & Qualitative \\
\hline
\end{tabular}

${ }^{\mathrm{a}}$ mHealth: mobile health.

${ }^{\mathrm{b}}$ EQ-5D VAS: European health-related quality of life utility with a visual analogue scale.

\section{Risk of Bias Within Studies}

At the study level, reviewers recorded observations of bias. The most frequently observed form of bias was selection bias (asking for volunteers for a research study involving technology will result in volunteers who already gravitate toward technology), which occurred in 7 out of 57 (13\%) articles analyzed [15,26,30-32,37,39]. There were six instances of convenience samples from a local population [34,49-52,64]. Both examples of bias limit the external validity of the results.

\section{Results of Individual Studies}

Themes that resulted from the narrative analysis are listed in Table 2. Repetition can be observed in a frame of a theme owing to multiple observations from the same article for that theme. Translations from observations to themes for interventions, medical outcomes, and barriers are listed in Multimedia Appendix 1, Multimedia Appendix 2, and Multimedia Appendix 3 , respectively. These appendices illustrate the logical inference reviewers made for each theme. For instance, one article listed remote patient monitoring for blood pressure, pulse oximeter, 
and body weight scales. These were categorized under telemonitoring [26]. The same article listed a decrease in hospital visits and a decrease in readmissions. These were categorized under an increase in hospital metrics. Additional data collected (bias, statistics, country of origin, and quality assessments) are displayed in Multimedia Appendix 4. In consensus meeting number two, we identified general observations, as depicted in the tables [20]. 
Table 2. Summary of the analysis of each article.

\begin{tabular}{|c|c|c|c|}
\hline Authors, year & Intervention & Medical outcome theme & Theme of barriers \\
\hline Hamilton et al, 2020 [26] & Telemonitoring & Increase in hospital metrics & Not reported \\
\hline \multirow[t]{3}{*}{ Theis et al, 2019 [5] } & \multirow[t]{3}{*}{ eHealth } & \multirow[t]{3}{*}{ Increase in satisfaction } & Medical literacy \\
\hline & & & Trust of the internet \\
\hline & & & Ownership of technology ${ }^{a}$ \\
\hline \multirow[t]{3}{*}{ Wildenbos et al, 2019 [27] } & \multirow[t]{3}{*}{ mHealth $^{\mathrm{b}}$} & \multirow[t]{3}{*}{ Increase in cognitive ability } & Visual acuity $^{\mathrm{a}}$ \\
\hline & & & Mental acuity \\
\hline & & & Technical literacy \\
\hline \multirow[t]{6}{*}{ Jakobsson et al, 2019 [28] } & mHealth & \multirow[t]{6}{*}{ Increase in cognitive ability } & Social implications \\
\hline & eHealth & & Privacy and security ${ }^{\mathrm{a}}$ \\
\hline & Telecare (phone) & & Technical literacy \\
\hline & & & Lack of desire \\
\hline & & & Ownership of technology \\
\hline & & & Lack of technical support \\
\hline \multirow[t]{5}{*}{ Karlsen et al, 2019 [29] } & \multirow[t]{5}{*}{ Telemonitoring } & Increase in safety or security & Mental acuity \\
\hline & & Increase in health-related quality of life & Visual acuity \\
\hline & & Increase in safety or security ${ }^{a}$ & Social implications \\
\hline & & Increase in autonomy & \\
\hline & & Increase in mindfulness of the condition & \\
\hline Coley et al, 2019 [30] & eHealth & Not reported & Trust of the internet \\
\hline \multirow{2}{*}{$\begin{array}{l}\text { Giesbrecht \& Miller, } 2019 \\
\text { [31] }\end{array}$} & \multirow[t]{2}{*}{ eHealth } & Increase in cognitive ability & \multirow[t]{2}{*}{ Not reported } \\
\hline & & Increase in safety or security & \\
\hline \multirow[t]{2}{*}{ Brodbeck et al, 2019 [32] } & \multirow[t]{2}{*}{ eHealth } & Decrease in psychological distress ${ }^{\mathrm{a}}$ & \multirow[t]{2}{*}{ Not reported } \\
\hline & & Increase in health-related quality of life & \\
\hline Mosley et al, 2019 [33] & eHealth & Not reported & Cost \\
\hline \multirow[t]{5}{*}{ Jensen et al, 2019 [34] } & \multirow[t]{5}{*}{ eHealth } & \multirow[t]{5}{*}{ Increase in autonomy ${ }^{\mathrm{a}}$} & Privacy and security \\
\hline & & & Ownership of technology \\
\hline & & & Lack of desire \\
\hline & & & Lack of technical support \\
\hline & & & Technical literacy \\
\hline \multirow[t]{3}{*}{ Rasche et al, 2018 [15] } & \multirow[t]{3}{*}{ eHealth } & \multirow[t]{3}{*}{ Not reported } & Trust of the internet \\
\hline & & & Technical literacy $^{\mathrm{a}}$ \\
\hline & & & Privacy and security \\
\hline \multirow[t]{2}{*}{ Portz et al, 2018 [35] } & \multirow[t]{2}{*}{ mHealth } & Increase in mindfulness of the condition & Technical literacy \\
\hline & & Increase in autonomy & Medical literacy \\
\hline Castro Sweet et al, 2018 [36] & eHealth & $\begin{array}{l}\text { Decrease in medical conditions surrounding dia- } \\
\text { betes }^{\text {a }}\end{array}$ & Not reported \\
\hline \multirow[t]{4}{*}{ Joe et al, 2018 [37] } & eHealth & Not reported & Visual acuity $^{\mathrm{a}}$ \\
\hline & & & Hand-eye coordination \\
\hline & & & Technical literacy \\
\hline & & & Lack of desire \\
\hline Dham et al, 2018 [4] & Telehealth video call & Increase in satisfaction & Visual acuity \\
\hline & & & Auditory acuity \\
\hline Paige et al, 2018 [16] & eHealth & Not reported & Technical literacy \\
\hline & & & Trust of the internet \\
\hline
\end{tabular}




\begin{tabular}{|c|c|c|c|}
\hline Authors, year & Intervention & Medical outcome theme & Theme of barriers \\
\hline Cajita et al, 2018 [38] & mHealth & Not reported & $\begin{array}{l}\text { Medical literacy } \\
\text { Mental acuity } \\
\text { Lack of desire } \\
\text { Technical literacy } \\
\text { Ownership of technology } \\
\text { Cost }\end{array}$ \\
\hline Harte et al, 2018 [39] & mHealth & Not reported & Technical literacy \\
\hline $\begin{array}{l}\text { Gordon \& Hornbrook, } 2018 \\
\text { [40] }\end{array}$ & eHealth & Not reported & $\begin{array}{l}\text { Cost } \\
\text { Technical literacy }\end{array}$ \\
\hline Bao et al, 2018 [41] & eHealth & $\begin{array}{l}\text { Increase in cognitive ability } \\
\text { Increase in activity or coordination }^{\mathrm{a}}\end{array}$ & Not reported \\
\hline Egede et al, 2018 [42] & Telehealth video call & $\begin{array}{l}\text { Decrease in psychological distress } \\
\text { Decrease in medical conditions surrounding pain }\end{array}$ & Not reported \\
\hline Platts-Mills et al, 2018 [43] & Telecare (phone) & Decrease in medical conditions surrounding pain & Not reported \\
\hline $\begin{array}{l}\text { Lopez-Villegas et al, } 2018 \\
\text { [44] }\end{array}$ & Telemonitoring & Increase in health-related quality of life & Not reported \\
\hline Dugas et al, 2018 [45] & mHealth & $\begin{array}{l}\text { Decrease in medical conditions surrounding dia- } \\
\text { betes }^{\text {a }}\end{array}$ & Not reported \\
\hline Nalder et al, 2018 [46] & eHealth & $\begin{array}{l}\text { Decrease in medical conditions surrounding dia- } \\
\text { betes } \\
\text { Increase in autonomy } \\
\text { Decrease in psychological distress } \\
\text { Increase in autonomy }\end{array}$ & Technical literacy \\
\hline Buck et al, 2017 [47] & eHealth & $\begin{array}{l}\text { Increase in documentation to give the provider } \\
\text { Increase in mindfulness of the condition }\end{array}$ & Technical literacy \\
\hline Ware et al, 2017 [14] & eHealth & Not reported & $\begin{array}{l}\text { Trust of the internet } \\
\text { Medical literacy } \\
\text { Technical literacy } \\
\text { Social implications } \\
\text { Lack of technical support } \\
\text { Privacy and security }\end{array}$ \\
\hline Chang et al, 2017 [48] & mHealth & Increase in autonomy ${ }^{\mathrm{a}}$ & Cost \\
\hline Cajita et al, 2017 [49] & mHealth & Not reported & $\begin{array}{l}\text { Medical literacy } \\
\text { Lack of desire } \\
\text { Cost } \\
\text { Technical literacy } \\
\text { Social implications }\end{array}$ \\
\hline LaMonica et al, 2017 [50] & eHealth & Increase in cognitive ability & $\begin{array}{l}\text { Auditory acuity } \\
\text { Cost } \\
\text { Auditory acuity }\end{array}$ \\
\hline Bahar-Fuchs et al, 2017 [51] & eHealth & $\begin{array}{l}\text { Increase in cognitive ability } \\
\text { Decrease in psychological distress }\end{array}$ & Not reported \\
\hline Nahm et al, 2017 [52] & eHealth & $\begin{array}{l}\text { Increase in mindfulness of the condition } \\
\text { Increase in autonomy } \\
\text { Increase in activity or coordination }\end{array}$ & Not reported \\
\hline
\end{tabular}




\begin{tabular}{|c|c|c|c|}
\hline Authors, year & Intervention & Medical outcome theme & Theme of barriers \\
\hline Knaevelsrud et al, 2017 [53] & eHealth & $\begin{array}{l}\text { Increase in safety or security } \\
\text { Increase in satisfaction } \\
\text { Increase in autonomy } \\
\text { Increase in health-related quality of life }\end{array}$ & Not reported \\
\hline Reijnders et al, 2017 [54] & eHealth & $\begin{array}{l}\text { Increase in activity or coordination } \\
\text { Increase in cognitive ability } \\
\text { Increase in autonomy }\end{array}$ & $\begin{array}{l}\text { Not reported } \\
\text { Privacy and security }\end{array}$ \\
\hline Hamblin et al, 2017 [56] & Telemonitoring & $\begin{array}{l}\text { Increase in autonomy } \\
\text { Increase in mindfulness of the condition } \\
\text { Increase in safety or security }\end{array}$ & $\begin{array}{l}\text { Technical literacy } \\
\text { Medical literacy } \\
\text { Social implications }^{\mathrm{a}}\end{array}$ \\
\hline Mageroski et al, 2016 [55] & Telemonitoring & Not reported & Cost \\
\hline Wang et al, 2016 [57] & Telemonitoring & Not reported & Lack of desire \\
\hline $\begin{array}{l}\text { Gordon \& Hornbrook, } 2016 \\
\text { [58] }\end{array}$ & eHealth & Not reported & $\begin{array}{l}\text { Ownership of technology } \\
\text { Lack of technical support } \\
\text { Cost } \\
\text { Technical literacy } \\
\text { Hand-eye coordination } \\
\text { Trust of the internet } \\
\text { Social implications } \\
\text { Lack of desire }\end{array}$ \\
\hline Williams et al, 2016 [59] & eHealth & Not reported & $\begin{array}{l}\text { Technical literacy } \\
\text { Lack of technical support } \\
\text { Mental acuity } \\
\text { Visual acuity } \\
\text { Hand-eye coordination }\end{array}$ \\
\hline Evans et al, 2016 [19] & mHealth & Increase in documentation to give the provider & $\begin{array}{l}\text { Lack of desire } \\
\text { Technical literacy } \\
\text { Lack of desire } \\
\text { Ownership of technology }\end{array}$ \\
\hline Muller et al, 2016 [60] & mHealth & $\begin{array}{l}\text { Increase in activity or coordination } \\
\text { Decrease in psychological distress } \\
\text { Decrease in medical conditions surrounding dia- } \\
\text { betes } \\
\text { Increase in mindfulness of the condition } \\
\text { Increase in guilt }\end{array}$ & Lack of desire \\
\hline Quinn et al, 2016 [61] & mHealth & $\begin{array}{l}\text { Decrease in medical conditions surrounding dia- } \\
\text { betes }\end{array}$ & $\begin{array}{l}\text { Visual acuity } \\
\text { Auditory acuity }\end{array}$ \\
\hline Royackers et al, 2016 [62] & eHealth & $\begin{array}{l}\text { Increase in safety or security } \\
\text { Increase in autonomy }{ }^{\mathrm{a}}\end{array}$ & Not reported \\
\hline Duh et al, 2016 [63] & Telecare (phone) & Not reported & $\begin{array}{l}\text { Mental acuity } \\
\text { Lack of desire } \\
\text { Lack of technical support } \\
\text { Technical literacy } \\
\text { Cost }\end{array}$ \\
\hline
\end{tabular}




\begin{tabular}{|c|c|c|c|}
\hline Authors, year & Intervention & Medical outcome theme & Theme of barriers \\
\hline Depatie \& Bigbee, 2015 [64] & mHealth & Not reported & $\begin{array}{l}\text { Cost } \\
\text { Lack of desire } \\
\text { Social implications } \\
\text { Technical literacy } \\
\text { Lack of technical support } \\
\text { Privacy and security }\end{array}$ \\
\hline Moore et al, 2015 [65] & eHealth & Not reported & $\begin{array}{l}\text { Technical literacy } \\
\text { Computer anxiety } \\
\text { Lack of technical support }\end{array}$ \\
\hline Currie et al, 2015 [66] & eHealth & Decrease in medical conditions surrounding pain & Social implications \\
\hline Grant et al, 2015 [67] & Telemonitoring & $\begin{array}{l}\text { Increase in health-related quality of life } \\
\text { Increase in autonomy }^{\mathrm{a}}\end{array}$ & $\begin{array}{l}\text { Lack of desire } \\
\text { Cost } \\
\text { Privacy and security }\end{array}$ \\
\hline Brenes et al, 2015 [68] & Telecare (phone) & Decrease in psychological distress ${ }^{\mathrm{a}}$ & Not reported \\
\hline Corbett et al, 2015 [69] & eHealth & $\begin{array}{l}\text { Increase in cognitive ability } \\
\text { Increase in health-related quality of life }\end{array}$ & Not reported \\
\hline Mavandadi et al, 2015 [70] & Telecare (phone) & Decrease in psychological distress ${ }^{\mathrm{a}}$ & Not reported \\
\hline Egede et al, 2015 [71] & Telehealth video call & Decrease in psychological distress ${ }^{\mathrm{a}}$ & Not reported \\
\hline Chang et al, 2015 [72] & Telemonitoring & Increase in mindfulness of the condition ${ }^{\mathrm{a}}$ & Not reported \\
\hline Boulos et al, 2015 [73] & eHealth & Increase in documentation to give the provider & $\begin{array}{l}\text { Medical literacy } \\
\text { Lack of technical support } \\
\text { Mental acuity } \\
\text { Hand-eye coordination } \\
\text { Visual acuity }\end{array}$ \\
\hline Dino \& deGuzman, 2015 [74] & $\begin{array}{l}\text { mHealth } \\
\text { eHealth } \\
\text { Telemonitoring }\end{array}$ & Not reported & $\begin{array}{l}\text { Lack of desire } \\
\text { Lack of technical support }\end{array}$ \\
\hline Czaja et al, 2015 [75] & Telemonitoring & $\begin{array}{l}\text { Increase in autonomy }{ }^{\mathrm{a}} \\
\text { Decrease in medical conditions surrounding dia- } \\
\text { betes }\end{array}$ & Technical literacy \\
\hline Choi et al, 2015 [76] & Telehealth video call & $\begin{array}{l}\text { Decrease in psychological distress } \\
\text { Increase in mindfulness of the condition } \\
\text { Increase in autonomy }\end{array}$ & $\begin{array}{l}\text { Ownership of technology } \\
\text { Lack of desire }\end{array}$ \\
\hline
\end{tabular}

${ }^{\mathrm{a}}$ Multiple uses of this theme in the same article. See Multimedia Appendix 1 for a complete list of individual observations and their translation to themes.

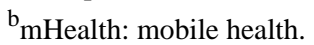

\section{Risk of Bias Across Studies and Quality Assessments}

Table 3 summarizes the quality indicators identified by the JHNEBP tool [15]. The most frequent strength rating was III, followed by I, II, and IV. The most frequent evidence rating was A, followed by B and C. No strengths below IV were encountered. A full list of quality assessments is presented in Multimedia Appendix 4. Articles that did not meet the minimum standards of quality were not included in the analysis. This decision was made to limit the bias inherent to nondata-driven opinions or conclusions that do not logically follow the data. 
Table 3. Summary of quality indicators.

\begin{tabular}{ll}
\hline Quality indicator & Value (N=57), n (\%) \\
\hline Strength of evidence & \\
I (experimental study, RCT ${ }^{\mathrm{a}}$, or meta-analysis of RCT) & $18(32 \%)$ \\
II (quasiexperimental study) & $10(17 \%)$ \\
III (nonexperimental, qualitative, or meta-synthesis study) & $28(49 \%)$ \\
IV (opinion) & $1(2 \%)$ \\
Quality of evidence & \\
A (high quality) & $33(58 \%)$ \\
B (good quality) & $23(40 \%)$ \\
C (low quality or major flaws) & $1(2 \%)$ \\
\hline
\end{tabular}

${ }^{\mathrm{a}} \mathrm{RCT}$ : randomized controlled trial.

\section{Additional Analysis}

The results of consensus meeting three identified the themes that corresponded with telehealth interventions, barriers to the use of telehealth, and medical outcomes. These are summarized in Tables 4-6.

\section{Interventions of Telehealth}

Five themes for interventions were identified. Two studies used multiple telehealth interventions. Table 4 lists the interventions with the associated references, number of occurrences, and probability of occurrence in the review. The most common intervention was eHealth (computer driven), followed by mHealth (smart device driven), telemonitoring (remote sensors), telecare (phone), and telehealth video call.

Table 4. Affinity matrix for telehealth interventions.

\begin{tabular}{llll}
\hline Intervention & References & $\begin{array}{l}\text { Number of occurrences } \\
(\mathrm{N}=62)\end{array}$ & $\begin{array}{l}\text { Probability of occurrence } \\
\text { eHealth }\end{array}$ \\
mHealth & {$[5,14-16,28,30-34,36,37,40,41,46,47,50-54,58,59,62,65,66,69,73,74]$} & 29 & $47 \%$ \\
Telemonitoring & {$[19,27,28,35,38,39,45,48,49,60,61,64,74]$} & 13 & $21 \%$ \\
Telecare (phone) & {$[26,29,44,55-57,67,72,74,75]$} & 10 & $16 \%$ \\
Video call & {$[4,29,42,71,76]$} & 5 & $8 \%$ \\
\hline
\end{tabular}

${ }^{a}$ mHealth: mobile health.

\section{Medical Outcomes and Health-Related Quality of Life Enhancers}

Thirteen themes and one observation that did not correspond with a theme for medical outcomes and quality of life factors were reported. Table 5 lists the outcomes with their associated references, number of occurrences, and probability of occurrence in this review. The most common theme for medical outcomes associated with telehealth interventions was decrease in psychological distress (decrease in anxiety symptoms, decrease in depressive symptoms, decrease in embitterment, decrease in grief, decrease in worry, decrease in loneliness, increase in emotional support, and increase in mood), with 21 of 118 (18\%) occurrences $[32,42,46,51,60,68,70,71,76]$. The theme associated with quality of life factors was listed as an increase in autonomy (increase in locus of control, increase in autonomy, increase in responsibility, increase in motivation to self-manage, and increase in independence), with 18 of 118 (15\%) occurrences $[29,34,35,46,48,52-54,56,62,67,75,76]$. One theme was associated with an increase in cognitive ability (increase in skill ability, increase in sensory organization, increase in memory, increase in cognitive activity, and increase in reasoning), with 11 of 118 (9\%) occurrences [19,20,23,32,41,42,60]. Another theme was associated with a decrease in symptoms surrounding diabetes (decrease in $\mathrm{HbA}_{1 \mathrm{c}}$, decrease in cholesterol, increase in glucose management, and increase in diabetes health), with 9 of $118(8 \%)$ occurrences $[28,36,37,51,52,66]$. Another theme was associated with an increase in mindfulness of the condition (increase in medical events detected, increase in education exposure, and more awareness of danger areas for falls like outside or stairwells), with 8 of 118 (7\%) occurrences $[21,27,38,43,47,51,63,67]$. The next theme summarized observations of an increase in the sense of safety, security, or comfort, with 7 of $118(6 \%)$ occurrences [21,23,44,47,53]. The last set of themes comprised $25 \%$ of the observations, and they were an increase in health-related quality of life (increase in life satisfaction and increase in the feeling of being understood); increase in activity or coordination (increase in mobility, increase in activity, increase in exercise, decrease in weight, decrease in BMI, increase in balance evaluation, and increase 
in the feeling of stability); decrease in medical conditions surrounding pain (decrease in alcohol abuse, decrease in cannabis misuse, decrease in cannabis dependence, and decrease in pain); increase in documentation to give to the provider (documentation and communication with the provider); increase in satisfaction (satisfaction with the health care system); and increase in hospital metrics (decrease in readmissions and decrease in hospital visits). The last observation was the only negative outcome. One participant noted that the SMS text messages she received as part of an exercise RCT increased her level of guilt because she was not exercising.

Table 5. Affinity matrix for medical outcomes and quality of life factors observed by older adults using telehealth.

\begin{tabular}{|c|c|c|c|}
\hline Theme or observation & References & $\begin{array}{l}\text { Number of occurrences } \\
(\mathrm{N}=118)\end{array}$ & Probability of occurrence \\
\hline Decrease in psychological distress & {$[32,42,46,51,60,68,70,71,76]$} & 21 & $19 \%$ \\
\hline Increase in autonomy & {$[29,34,35,46,48,52-54,56,62,67,75,76]$} & 18 & $16 \%$ \\
\hline Not reported & {$[14-16,30,33,37-40,49,55,57-59,63-65,74]$} & 18 & $16 \%$ \\
\hline Increase in cognitive ability & {$[27,28,31,41,50,51,54,69]$} & 11 & $10 \%$ \\
\hline $\begin{array}{l}\text { Decrease in medical conditions surrounding } \\
\text { diabetes }\end{array}$ & {$[36,45,46,60,61,75]$} & 9 & $8 \%$ \\
\hline Increase in mindfulness of the condition & {$[29,35,47,52,56,60,72,77]$} & 8 & $7 \%$ \\
\hline Increase in safety or security & {$[29,31,53,56,62]$} & 7 & $6 \%$ \\
\hline Increase in health-related quality of life & {$[29,32,44,53,67,69]$} & 6 & $5 \%$ \\
\hline Increase in activity or coordination & {$[41,52,54,60]$} & 6 & $5 \%$ \\
\hline $\begin{array}{l}\text { Decrease in medical conditions surrounding } \\
\text { pain }\end{array}$ & {$[42,43,66]$} & 5 & $4 \%$ \\
\hline $\begin{array}{l}\text { Increase in documentation to give the } \\
\text { provider }\end{array}$ & {$[19,47,73]$} & 3 & $3 \%$ \\
\hline Increase in satisfaction & {$[4,5,53]$} & 3 & $3 \%$ \\
\hline Increase in hospital metrics & {$[26]$} & 2 & $2 \%$ \\
\hline Increase in guilt & {$[60]$} & 1 & $1 \%$ \\
\hline
\end{tabular}

\section{Barriers}

Fourteen themes and one observation that did not fit into a theme for barriers were observed. Table 6 lists the barriers with their associated references, number of occurrences, and probability of occurrence in this review. The barrier that was reported most often was technical literacy (I do not understand technology, I cannot navigate menus, I do not know how, etc) [14-16,19,27,28,34,35,37-40,46,47,49,56,58,59,63-65,75]. The theme noted the second most often was lack of desire (laziness, I do not want to, I am too busy, etc) [19,28,34,37, $38,49,57,58,60,63,64,67,74,76]$. Another theme was cost (too expensive, we live off a fixed income, etc) [33,38, $40,48-50,55,58,63,64,67]$. The theme lack of technical support included the following: my friends or family are not able to help me, I do not understand the interface, etc [14,28,34,58,63-65,73,74]. The theme visual acuity included the following: fonts or icons are too small, color contrast, etc $[4,27,29,37,59,61,73]$. The next observation was a surprise to our reviewing team; the theme was social implications of using a telemonitoringdevice (I do not want to bother a first responder, I do not want a stranger coming to my house, I do not want anyone coming to my house late at night, I had a bad experience the last time I used the telemonitoring device, I do not want my neighbor to overhear me using this thing, I do not have my own email, I do not understand social media, etc) $[14,28,29,49,56,58,64,66]$. The next theme was ownership of technology (no phone, no computer, no internet access, etc) $[5,19,28,34,38,58,76]$. The last set of themes and observations comprised less than $25 \%$ of the observations, and they were privacy and security concerns, medical literacy (I do not understand terminology, I do not understand test results, etc), trust of the internet, mental acuity (computers confuse me, the interface is too complex, I cannot focus for very long, how did I get to this page? etc), hand-eye coordination (particularly with those who have Parkinson disease, but not exclusively), auditory acuity, and computer anxiety. 
Table 6. Affinity matrix for barriers to the use of telehealth by older adults.

\begin{tabular}{llll}
\hline Themes of barriers & References & $\begin{array}{l}\text { Number of occurrences } \\
(\mathrm{N}=144)\end{array}$ & Probability of occurrence \\
\hline Technical literacy & {$[14-16,19,27,28,34,35,37-40,46,47,49,56,58,59,63-65,75]$} & 25 & $17 \%$ \\
Not reported & {$[26,31,32,36,41-45,51-54,62,68-72]$} & 19 & $13 \%$ \\
Lack of desire & {$[19,28,34,37,38,49,57,58,60,63,64,67,74,76]$} & 15 & $10 \%$ \\
Cost & {$[33,38,40,48-50,55,58,63,64,67]$} & 11 & $8 \%$ \\
Lack of technical support & {$[14,28,34,58,63-65,73,74]$} & 10 & $7 \%$ \\
Visual acuity & {$[4,27,29,37,59,61,73]$} & 10 & $7 \%$ \\
Social implications & {$[14,28,29,49,56,58,64,66]$} & 9 & $6 \%$ \\
Ownership of technology & {$[5,19,28,34,38,58,76]$} & 8 & $6 \%$ \\
Privacy and security & {$[5,19,28,34,38,58,76]$} & 8 & $6 \%$ \\
Medical literacy & {$[5,14,15,35,38,49,56,73]$} & 8 & $6 \%$ \\
Trust of the internet & {$[5,14-16,30,58]$} & 6 & $4 \%$ \\
Mental acuity & {$[27,29,38,59,63,73]$} & 6 & $4 \%$ \\
Hand-eye coordination & {$[37,58,59,73]$} & 4 & $3 \%$ \\
Auditory acuity & {$[4,50,61]$} & 4 & $3 \%$ \\
Computer anxiety & {$[65]$} & 1 & $1 \%$ \\
\hline
\end{tabular}

\section{Interactions Between Observations}

There were several interactions worth discussing. We analyzed the interactions between interventions and barriers. Ten instances of eHealth interventions were mentioned with technical literacy [14-16,30,33,37,40,58,59,65,74]. Eight instances of eHealth interventions were mentioned with lack of technical support $[14,28,34,58,59,65,73,74]$. There were eight instances of mHealth interventions associated with technical literacy [19,27,28,35,38,39,49,64], but these were hardly mentioned at all with lack of technical support [28,74]. The interventions of mHealth were also associated with the barrier of lack of desire. This occurred six times in the literature $[28,38,39,60,64,74]$. Contrary to literature on the digital divide, eHealth and mHealth were only marginally associated with ownership of technology, which occurred four [5,28,34,58] and three times $[19,28,38]$, respectively. Commensurate with literature on generational trends, both eHealth and mHealth were associated with privacy and security concerns, which occurred four [14,15,34,35] and two times [28,64], respectively. Both eHealth and mHealth were associated with the barrier medical literacy, which occurred four $[5,14,15,73]$ and three times [35,38,39], respectively. Surprisingly, eHealth was associated with hand-eye coordination, but mHealth was not [37,58,60,73]. Finally, eHealth was associated with lack of trust of the internet, which occurred six times in the literature [5,14-16,30,58].

We also analyzed the interactions between interventions and medical outcomes. eHealth interventions were associated with an increase in cognitive ability. This interaction occurred seven times in the literature [28,31,41,50,51,54,69].

\section{Results Summary}

This review identified 13 themes and one lone observation of medical outcomes incident with the adoption of five types of telehealth approaches. This review also identified 14 themes and one observation of barriers to the adoption of telehealth.

\section{Discussion}

\section{Common Barriers to Telehealth}

In this review, we were able to identify the common barriers associated with older adults utilizing telehealth. The most frequent barriers were lack of desire, cost, lack of technical support, visual acuity, social implications of use, ownership of technology, privacy and security, medical literacy, trust of the internet, mental acuity, hand-eye coordination, auditory acuity, and computer anxiety. Each of these barrier areas could present hurdles for elderly people dealing with telehealth and reasons to not use it. Lack of technical literacy is a large area of concern, as many elderly people have issues using computers to check email or smartphones to make telephone calls [13]. Because this is new to this population, they are also being held back from acceptance by a simple lack of wanting to do it $[28,34,37-39,57,58,60,63,64,67,74,76]$. It seems to be an easy thing to add to one's daily tasks, but when one has lived largely without the use of these technologies, it can become an arduous task to "sell" the benefits of the sudden use of new technology and learning how to use new technology. They have the attitude "as it was not needed before, why bother to learn it now?" This can prove to be an uphill battle for providers who are attempting to utilize new technologies in different ways.

The cost of technology is also quite prohibitive, as computers, smartphones, and other devices cost hundreds to thousands of dollars. Those living on fixed incomes are cash strapped and may not be able to afford to purchase or use such new technologies. Not owning such technologies presents its own concerns for the provision of care. Besides cost, there are concerns in this population regarding the ability to actually 
utilize the modality of telehealth efficiently. Issues with visual acuity $[4,27,29,37,59,61,73]$, mental acuity $[27,29,38,59,63,73]$, hand-eye coordination $[37,58,59,73]$, and auditory acuity $[4,50,61]$ are all relevant concerns for elderly people. Many people, as they become older, experience decreases in the efficiencies of the operations of many body systems, including their senses. Many develop disease processes that can affect their mental status, vision, and hearing, and any or all of these could easily lead to problems with being able to use technology, let alone having a clear understanding of what they need to be doing with the device or even how to interact with it.

The elderly population also has relevant concerns with trust and technology, as they are one of the prime targets for abuse from their use of technology according to popular media $[13,78]$. This is where lack of technical support for the use of technology can become a very relevant area of concern. There is no affordable and adequate source of "technical support" to simply learn how to use devices $[14,28,59,73]$. This lack of knowledge and available education can be a very problematic barrier for the use of the modality of telehealth. Furthermore, problems surrounding trust of the internet [5,14-16,30,58], concerns of privacy and security $[5,19,28,34,38,58,76]$, and even computer anxiety [65] can figure into the use of technology. As there are concerns with privacy and security, telehealth could easily cause patients to succumb to some level of anxiety. Not understanding the modality of telehealth or how to use it can add to the level of this anxiety at an exponential rate.

Another consideration with the use of telehealth is that it requires a certain level of user knowledge. The utilization of medical applications requires the user to have some knowledge of medical terms, procedures, etc $[5,14,15,35,38,49,56,73]$. This is often not the case, as this population was raised without the internet or medical knowledge. Medical knowledge came from physicians during their younger years, and only recently, the approach has changed to the utilization of internet web searches to garner knowledge about symptoms and diagnoses. This is an entirely new world for the elderly population and a relevant barrier to the use of these applications overall. Overcoming this knowledge gap could prove to be an insurmountable task or one that requires any telehealth use to be kept to an absolute minimum for knowledge or know-how on the part of the user.

\section{Common Outcomes Associated With Telehealth Interventions}

The research supports strong medical outcomes incident to the use of telehealth as follows: decreased psychological distress $[32,42,46,51,60,68,70,71,76], \quad$ increased autonomy $[29,34,35,46,48,52-54,56,62,67,75,76]$, increased cognitive ability [27,28,31,41,50,51,54,69], and many others. This review supports an increased quality of life for those who adopt telehealth $[29,32,44,53,67,69]$. The use of telehealth can lead to less psychological distress, as users know that they have a way of communicating their medical concerns to their providers in a much easier and faster way. This could eventually enable better health due to better management, thus allowing for fewer associated medical conditions for those patients who use telehealth for assistance in the management of their care.
The observation of greater documentation for providers demonstrated that the use of telehealth is not all about the patient. It is just as much about practitioners providing care. The use of telehealth allows for much faster accessibility to documentation to provide care or even real-time information about the patient to allow for immediate diagnosis or intervention, based on information being gathered by the used technology. This can make the provision of care easier and much more efficient for the field, which is already seeing more patients than it can comfortably manage.

\section{Interactions Among Outcomes, Barriers, and Types of Interventions}

eHealth interventions were the most frequently observed interventions in the literature, and these interventions were most frequently associated with the barriers of technical literacy and lack of technical support. This observation is interesting because general technical support, whether from friends, neighbors, family, or caregivers, or professionally acquired technical support is a control for the barrier of technical literacy. The interaction between eHealth and technical literacy is interesting as well. This could signal that older adults are more adept at mobile technology than computer technology for application of telehealth. This supposition is supported by the literature because many older adults are turning to mobile technology to communicate with children and grandchildren [13]. The interaction between mHealth and lack of desire is noteworthy. This seems to indicate that older adults are willing to interact with mobile technology to communicate with children and grandchildren, but they are not as willing to use it for telehealth interventions.

\section{Study Quality and Literature Bias}

The assessment of the quality of the articles studied is worthy of discussion. The majority $(27 / 57,49 \%)$ of the articles analyzed were level III (nonexperimental, qualitative, or meta-synthesis studies). The reviewers would have preferred to analyze only the highest level (level I) (experimental study or RCT), but only $10(17 \%)$ such studies were available. Fortunately, 98\% (56/57) of the articles were rated as quality level A (high quality) or B (good quality). The importance of this rating cannot be understated. If the findings from this review were from low-quality articles, the results would not be as strong. By analyzing high-quality articles with strong levels of evidence, readers can be more assured of the results. Research articles with strong study designs and sufficiently large samples are generally accepted in the scientific field for their veracity.

\section{Limitations}

The authors identified the low number of articles analyzed as a limitation of this systematic review. If the authors conduct another systematic review on the same topic, they would like to have a larger analysis pool. This could be achieved by broadening the years of study in the selection or by reducing the threshold of quality. However, the additional years of study would only repeat the results from previously published reviews of a similar topic, and lowering the threshold of quality would introduce articles with dubious results. 
Although not intentional, the authors realized that selection bias may be present in this article. To combat selection bias, the authors worked to minimize its effects by ensuring each article was reviewed by at least two authors. The authors held consensus meetings after each screening to provide feedback and reach total agreement on the inclusion and exclusion of articles for the analysis.

Another source of bias that could have affected this article is publication bias. To control for publication bias, the authors searched the Boolean search string in Google Scholar. This action was intended to identify articles from lesser-known journals that may not have appeared in MEDLINE or CINAHL.

Another limitation is our inclusion of people aged 50 years or above in the study of older adults. Most studies categorize older adults as those aged 65 years or above. The elderly population currently spans baby boomers and the silent generation. The youngest members of the former group are still working and are most likely using technology fluently. It is possible that our generalizations do not apply to all members of the elderly demographic.

\section{Future Research}

Health care systems can utilize knowledge of these barriers to develop solutions for broadening the use of telehealth among older adults. A multidisciplinary approach and culture of collaboration between administrative leadership and providers may be the most effective and immediate manner of implementing solutions to breach these barriers and strengthen the reach of health care services. However, some barriers may be out of the scope of impact, and policy makers should consider supporting the efforts. Future research should be conducted on methods for personalizing telehealth in older adults before implementation.

\section{Conclusion}

Providing sufficient health care access to the rapidly growing aging population has been an imminent issue, and telehealth is a useful tool that can provide a solution. While health care systems increase their telehealth efforts to improve access to health care services among vulnerable populations, such as older adults, some health care organizations do not consider the technological, educational, financial, and behavioral barriers before implementing telehealth solutions. It is imperative that health care systems use a multidisciplinary approach and collaborate with health care providers, community partners, and policy makers to address these barriers of utilizing telehealth among older adults and to successfully implement telehealth solutions. This systematic review provides some understanding of older adults' perspectives and experiences with the barriers of implementing telehealth services.

\section{Authors' Contributions}

Conceptualization: CSK, NW, JF, ENP, and SZ; methodology: CSK, NW, JF, ENP, and SZ; formal analysis: CSK, NW, JF, ENP, SZ, and MM; investigation: CSK, NW, JF ENP, and SZ; writing-original draft preparation: NW, JF, ENP, and SZ; writing-review and editing: JF; visualization: ENP; supervision: NW; project administration: SZ; final draft: CSK and MM.

\section{Conflicts of Interest}

None declared.

\section{Multimedia Appendix 1}

Detailed observations on interventions and corresponding themes.

[DOCX File, 29 KB-Multimedia Appendix 1]

\section{Multimedia Appendix 2}

Detailed observations on medical outcomes and corresponding themes.

[DOCX File, 42 KB-Multimedia Appendix 2]

\section{Multimedia Appendix 3}

Detailed observations on barriers and corresponding themes.

[DOCX File, 37 KB-Multimedia Appendix 3]

\section{Multimedia Appendix 4}

Bias, country of origin, statistics, and quality assessments.

[DOCX File , $51 \mathrm{~KB}-$ Multimedia Appendix 4]

\section{References}

1. Ageing and health. World Health Organization. 2018 Feb 05. URL: https://www.who.int/news-room/fact-sheets/detail/ ageing-and-health [accessed 2020-04-02]

2. From Pyramid to Pillar: A Century of Change, Population of the U.S. US Census Bureau. 2018 Mar 13. URL: https://www. census.gov/library/visualizations/2018/comm/century-of-change.html [accessed 2020-04-02] 
3. Colby S, Ortman J. The Baby Boom Cohort in the United States: 2012-2060. US Census Bureau. 2014 May 01. URL: https://www.census.gov/library/publications/2014/demo/p25-1141.html [accessed 2020-04-02]

4. Dham P, Gupta N, Alexander J, Black W, Rajji T, Skinner E. Community based telepsychiatry service for older adults residing in a rural and remote region- utilization pattern and satisfaction among stakeholders. BMC Psychiatry 2018 Sep 27;18(1):316 [FREE Full text] [doi: 10.1186/s12888-018-1896-3] [Medline: $\underline{\text { 30261845] }}$

5. Theis S, Schäfer D, Bröhl C, Schäfer K, Rasche P, Wille M, et al. Predicting technology usage by health information need of older adults: Implications for eHealth technology. Work 2019;62(3):443-457. [doi: 10.3233/WOR-192878] [Medline: 30909259]

6. Ryu S. Telemedicine: Opportunities and Developments in Member States: Report on the Second Global Survey on eHealth 2009 (Global Observatory for eHealth Series, Volume 2). Healthc Inform Res 2012;18(2):153. [doi: 10.4258/hir.2012.18.2.153]

7. Yoon H, Jang Y, Vaughan PW, Garcia M. Older Adults' Internet Use for Health Information: Digital Divide by Race/Ethnicity and Socioeconomic Status. J Appl Gerontol 2020 Jan;39(1):105-110. [doi: 10.1177/0733464818770772] [Medline: 29661052]

8. Dorsey ER, Topol EJ. Telemedicine 2020 and the next decade. The Lancet 2020 Mar;395(10227):859. [doi: 10.1016/s0140-6736(20)30424-4]

9. Trump Administration Issues Second Round of Sweeping Changes to Support U.S. Healthcare System During COVID-19 Pandemic. Centers for Medicare \& Medicaid Services. 2020 Apr 30. URL: https://www.cms.gov/newsroom/press-releases/ trump-administration-issues-second-round-sweeping-changes-support-us-healthcare-system-during-covid [accessed 2020-04-30]

10. Foster MV, Sethares KA. Facilitators and barriers to the adoption of telehealth in older adults: an integrative review. Comput Inform Nurs 2014 Nov;32(11):523-33; quiz 534. [doi: 10.1097/CIN.0000000000000105] [Medline: 25251862]

11. Kampmeijer R, Pavlova M, Tambor M, Golinowska S, Groot W. The use of e-health and m-health tools in health promotion and primary prevention among older adults: a systematic literature review. BMC Health Serv Res 2016 Sep 05;16 Suppl 5:290 [FREE Full text] [doi: 10.1186/s12913-016-1522-3] [Medline: 27608677]

12. Kruse CS, Krowski N, Rodriguez B, Tran L, Vela J, Brooks M. Telehealth and patient satisfaction: a systematic review and narrative analysis. BMJ Open 2017 Aug 03;7(8):e016242 [FREE Full text] [doi: 10.1136/bmjopen-2017-016242] [Medline: 28775188]

13. Kruse CS, Mileski M, Moreno J. Mobile health solutions for the aging population: A systematic narrative analysis. J Telemed Telecare 2017 May;23(4):439-451. [doi: 10.1177/1357633X16649790] [Medline: 27255207]

14. Ware P, Bartlett SJ, Paré G, Symeonidis I, Tannenbaum C, Bartlett G, et al. Using eHealth Technologies: Interests, Preferences, and Concerns of Older Adults. Interact J Med Res 2017 Mar 23;6(1):e3 [FREE Full text] [doi: 10.2196/ijmr.4447] [Medline: 28336506]

15. Rasche P, Wille M, Bröhl C, Theis S, Schäfer K, Knobe M, et al. Prevalence of Health App Use Among Older Adults in Germany: National Survey. JMIR Mhealth Uhealth 2018 Jan 23;6(1):e26 [FREE Full text] [doi: 10.2196/mhealth.8619] [Medline: 29362211]

16. Paige SR, Miller MD, Krieger JL, Stellefson M, Cheong J. Electronic Health Literacy Across the Lifespan: Measurement Invariance Study. J Med Internet Res 2018 Jul 09;20(7):e10434 [FREE Full text] [doi: 10.2196/10434] [Medline: 29986848]

17. Choi N. Telehealth Depression Treatments for Older Adults. ClinicalTrials.gov. 2015 Nov 09. URL: https://clinicaltrials. gov/ct2/show/NCT02600754 [accessed 2020-04-02]

18. Scogin F, Lichstein K, DiNapoli EA, Woosley J, Thomas SJ, LaRocca MA, et al. Effects of Integrated Telehealth-Delivered Cognitive-Behavioral Therapy for Depression and Insomnia in Rural Older Adults. J Psychother Integr 2018 Sep;28(3):292-309 [FREE Full text] [doi: 10.1037/int0000121] [Medline: 30930607]

19. Evans J, Papadopoulos A, Silvers CT, Charness N, Boot WR, Schlachta-Fairchild L, et al. Remote Health Monitoring for Older Adults and Those with Heart Failure: Adherence and System Usability. Telemed J E Health 2016 Jun;22(6):480-488 [FREE Full text] [doi: 10.1089/tmj.2015.0140] [Medline: 26540369]

20. Kruse CS. Writing a Systematic Review for Publication in a Health-Related Degree Program. JMIR Res Protoc 2019 Oct 14;8(10):e15490 [FREE Full text] [doi: 10.2196/15490] [Medline: $\underline{\text { 31527018] }}$

21. Moher D, Liberati A, Tetzlaff J, Altman DG. Preferred reporting items for systematic reviews and meta-analyses: the PRISMA statement. BMJ 2009 Jul 21;339:b2535. [doi: 10.1136/bmj.b2535] [Medline: 19621072]

22. Braun V, Clarke V. Using thematic analysis in psychology. Qualitative Research in Psychology 2006 Jan;3(2):77-101. [doi: 10.1191/1478088706qp063oa]

23. Dang D, Dearholt S. Johns Hopkins Nursing Evidence-based Practice: Model and Guidelines. Indianapolis, IN: SIGMA Theta Tau International; 2018.

24. Light RJ. Measures of response agreement for qualitative data: Some generalizations and alternatives. Psychological Bulletin 1971;76(5):365-377. [doi: 10.1037/h0031643]

25. McHugh ML. Interrater reliability: the kappa statistic. Biochem Med (Zagreb) 2012;22(3):276-282 [FREE Full text] [Medline: 23092060] 
26. Hamilton T, Johnson L, Quinn BT, Coppola J, Sachs D, Migliaccio J, et al. Telehealth Intervention Programs for Seniors: An Observational Study of a Community-Embedded Health Monitoring Initiative. Telemed J E Health 2020 Apr;26(4):438-445. [doi: 10.1089/tmj.2018.0248] [Medline: 30994409]

27. Wildenbos GA, Jaspers MW, Schijven MP, Dusseljee-Peute LW. Mobile health for older adult patients: Using an aging barriers framework to classify usability problems. Int J Med Inform 2019 Apr;124:68-77. [doi:

10.1016/j.ijmedinf.2019.01.006] [Medline: 30784429]

28. Jakobsson E, Nygård L, Kottorp A, Malinowsky C. Experiences from using eHealth in contact with health care among older adults with cognitive impairment. Scand J Caring Sci 2019 Jun;33(2):380-389. [doi: 10.1111/scs.12634] [Medline: $\underline{\text { 30628736] }}$

29. Karlsen C, Moe CE, Haraldstad K, Thygesen E. Caring by telecare? A hermeneutic study of experiences among older adults and their family caregivers. J Clin Nurs 2019 Apr;28(7-8):1300-1313. [doi: 10.1111/jocn.14744] [Medline: $\underline{30552788]}$

30. Coley N, Rosenberg A, van Middelaar T, Soulier A, Barbera M, Guillemont J, MIND-AD, HATICE groups. Older Adults' Reasons for Participating in an eHealth Prevention Trial: A Cross-Country, Mixed-Methods Comparison. J Am Med Dir Assoc 2019 Jul;20(7):843-849.e5. [doi: 10.1016/j.jamda.2018.10.019] [Medline: 30541689]

31. Giesbrecht EM, Miller WC. Effect of an mHealth Wheelchair Skills Training Program for Older Adults: A Feasibility Randomized Controlled Trial. Arch Phys Med Rehabil 2019 Nov;100(11):2159-2166. [doi: 10.1016/j.apmr.2019.06.010] [Medline: 31336101]

32. Brodbeck J, Berger T, Biesold N, Rockstroh F, Znoj HJ. Evaluation of a guided internet-based self-help intervention for older adults after spousal bereavement or separation/divorce: A randomised controlled trial. J Affect Disord 2019 Jun 01;252:440-449. [doi: 10.1016/j.jad.2019.04.008] [Medline: 31003114]

33. Mosley CL, Langley LM, Davis A, McMahon CM, Tremblay KL. Reliability of the Home Hearing Test: Implications for Public Health. J Am Acad Audiol 2019 Mar;30(3):208-216 [FREE Full text] [doi: 10.3766/jaaa.17092] [Medline: 30461396]

34. Jensen CM, Overgaard S, Wiil UK, Clemensen J. Can Tele-Health Support Self-Care and Empowerment? A Qualitative Study of Hip Fracture Patients' Experiences With Testing an “App”. SAGE Open Nursing 2019 Feb 21;5:237796081982575 [FREE Full text] [doi: 10.1177/2377960819825752]

35. Portz JD, Vehovec A, Dolansky MA, Levin JB, Bull S, Boxer R. The Development and Acceptability of a Mobile Application for Tracking Symptoms of Heart Failure Among Older Adults. Telemed J E Health 2018 Feb;24(2):161-165 [FREE Full text] [doi: 10.1089/tmj.2017.0036] [Medline: 28696832]

36. Castro Sweet CM, Chiguluri V, Gumpina R, Abbott P, Madero EN, Payne M, et al. Outcomes of a Digital Health Program With Human Coaching for Diabetes Risk Reduction in a Medicare Population. J Aging Health 2018 Jun;30(5):692-710 [FREE Full text] [doi: 10.1177/0898264316688791] [Medline: 28553807]

37. Joe J, Hall A, Chi N, Thompson H, Demiris G. IT-based wellness tools for older adults: Design concepts and feedback. Inform Health Soc Care 2018 Mar;43(2):142-158. [doi: 10.1080/17538157.2017.1290637] [Medline: 28350186]

38. Cajita MI, Hodgson NA, Lam KW, Yoo S, Han H. Facilitators of and Barriers to mHealth Adoption in Older Adults With Heart Failure. Comput Inform Nurs 2018 Aug;36(8):376-382 [FREE Full text] [doi: 10.1097/CIN.0000000000000442] [Medline: 29742549]

39. Harte R, Hall T, Glynn L, Rodríguez-Molinero A, Scharf T, Quinlan LR, et al. Enhancing Home Health Mobile Phone App Usability Through General Smartphone Training: Usability and Learnability Case Study. JMIR Hum Factors 2018 Apr 26;5(2):e18 [FREE Full text] [doi: 10.2196/humanfactors.7718] [Medline: 29699969]

40. Gordon NP, Hornbrook MC. Older adults' readiness to engage with eHealth patient education and self-care resources: a cross-sectional survey. BMC Health Serv Res 2018 Mar 27;18(1):220 [FREE Full text] [doi: 10.1186/s12913-018-2986-0] [Medline: 29587721]

41. Bao T, Carender WJ, Kinnaird C, Barone VJ, Peethambaran G, Whitney SL, et al. Effects of long-term balance training with vibrotactile sensory augmentation among community-dwelling healthy older adults: a randomized preliminary study. J Neuroeng Rehabil 2018 Jan 18;15(1):5 [FREE Full text] [doi: 10.1186/s12984-017-0339-6] [Medline: 29347946]

42. Egede LE, Walker RJ, Payne EH, Knapp RG, Acierno R, Frueh BC. Effect of psychotherapy for depression via home telehealth on glycemic control in adults with type 2 diabetes: Subgroup analysis of a randomized clinical trial. J Telemed Telecare 2018 Oct;24(9):596-602. [doi: 10.1177/1357633X17730419] [Medline: 28945160]

43. Platts-Mills TF, Hollowell AG, Burke GF, Zimmerman S, Dayaa JA, Quigley BR, et al. Randomized controlled pilot study of an educational video plus telecare for the early outpatient management of musculoskeletal pain among older emergency department patients. Trials 2018 Jan 05;19(1):10 [FREE Full text] [doi: 10.1186/s13063-017-2403-8] [Medline: 29304831]

44. Lopez-Villegas A, Catalan-Matamoros D, Lopez-Liria R, Enebakk T, Thunhaug H, Lappegård KT. Health-related quality of life on tele-monitoring for users with pacemakers 6 months after implant: the NORDLAND study, a randomized trial. BMC Geriatr 2018 Sep 21;18(1):223 [FREE Full text] [doi: 10.1186/s12877-018-0911-3] [Medline: $\underline{30241511]}$

45. Dugas M, Crowley K, Gao GG, Xu T, Agarwal R, Kruglanski AW, et al. Individual differences in regulatory mode moderate the effectiveness of a pilot mHealth trial for diabetes management among older veterans. PLoS One 2018;13(3):e0192807 [FREE Full text] [doi: 10.1371/journal.pone.0192807] [Medline: 29513683] 
46. Nalder E, Marziali E, Dawson DR, Murphy K. Delivering cognitive behavioural interventions in an internet-based healthcare delivery environment. British Journal of Occupational Therapy 2018 Mar 23;81(10):591-600. [doi: $10.1177 / 0308022618760786]$

47. Buck H, Pinter A, Poole E, Boehmer J, Foy A, Black S, et al. Evaluating the older adult experience of a web-based, tablet-delivered heart failure self-care program using gerontechnology principles. Geriatr Nurs 2017;38(6):537-541. [doi: 10.1016/j.gerinurse.2017.04.001] [Medline: 28554497]

48. Chang C, Lee T, Mills ME. Experience of Home Telehealth Technology in Older Patients With Diabetes. Comput Inform Nurs 2017 Oct;35(10):530-537. [doi: 10.1097/CIN.0000000000000341] [Medline: 28291156]

49. Cajita MI, Hodgson NA, Budhathoki C, Han H. Intention to Use mHealth in Older Adults With Heart Failure. J Cardiovasc Nurs 2017;32(6):E1-E7 [FREE Full text] [doi: 10.1097/JCN.0000000000000401] [Medline: 28248747]

50. LaMonica HM, English A, Hickie IB, Ip J, Ireland C, West S, et al. Examining Internet and eHealth Practices and Preferences: Survey Study of Australian Older Adults With Subjective Memory Complaints, Mild Cognitive Impairment, or Dementia. J Med Internet Res 2017 Oct 25;19(10):e358 [FREE Full text] [doi: 10.2196/jmir.7981] [Medline: 29070481]

51. Bahar-Fuchs A, Webb S, Bartsch L, Clare L, Rebok G, Cherbuin N, et al. Tailored and Adaptive Computerized Cognitive Training in Older Adults at Risk for Dementia: A Randomized Controlled Trial. J Alzheimers Dis 2017;60(3):889-911. [doi: 10.3233/JAD-170404] [Medline: 28922158]

52. Nahm E, Resnick B, Brown C, Zhu S, Magaziner J, Bellantoni M, et al. The Effects of an Online Theory-Based Bone Health Program for Older Adults. J Appl Gerontol 2017 Sep;36(9):1117-1144 [FREE Full text] [doi: 10.1177/0733464815617284] [Medline: 26675352]

53. Knaevelsrud C, Böttche M, Pietrzak RH, Freyberger HJ, Kuwert P. Efficacy and Feasibility of a Therapist-Guided Internet-Based Intervention for Older Persons with Childhood Traumatization: A Randomized Controlled Trial. Am J Geriatr Psychiatry 2017 Aug;25(8):878-888. [doi: 10.1016/j.jagp.2017.02.024] [Medline: 28365000]

54. Reijnders JS, Geusgens CA, Ponds RW, van Boxtel MP. "Keep your brain fit!" Effectiveness of a psychoeducational intervention on cognitive functioning in healthy adults: A randomised controlled trial. Neuropsychol Rehabil 2017 Jun;27(4):455-471. [doi: 10.1080/09602011.2015.1090458] [Medline: 26414279]

55. Mageroski A, Alsadoon A, Prasad P, Pham L, Elchouemi A. Impact of wireless communications technologies on elder people healthcare: Smart home in Australia. 2016 Presented at: 13th International Joint Conference on Compouter Science and Software Engineering (JCSSE); 2016; Khon Kaen, Thailand p. 1-6. [doi: 10.1109/jcsse.2016.7748862]

56. Hamblin K, Yeandle S, Fry G. Researching telecare: the importance of context. Journal of Enabling Technologies 2017 Sep 18;11(3):75-84. [doi: 10.1108/jet-04-2017-0016]

57. Wang J, Carroll D, Peck M, Myneni S, Gong Y. Mobile and Wearable Technology Needs for Aging in Place: Perspectives from Older Adults and Their Caregivers and Providers. Stud Health Technol Inform 2016;225:486-490. [Medline: 27332248]

58. Gordon NP, Hornbrook MC. Differences in Access to and Preferences for Using Patient Portals and Other eHealth Technologies Based on Race, Ethnicity, and Age: A Database and Survey Study of Seniors in a Large Health Plan. J Med Internet Res 2016 Mar 04;18(3):e50 [FREE Full text] [doi: 10.2196/jmir.5105] [Medline: 26944212]

59. Williams K, Pennathur P, Bossen A, Gloeckner A. Adapting Telemonitoring Technology Use for Older Adults: A Pilot Study. Res Gerontol Nurs 2016;9(1):17-23 [FREE Full text] [doi: 10.3928/19404921-20150522-01] [Medline: 26020575]

60. Müller AM, Khoo S, Morris T. Text Messaging for Exercise Promotion in Older Adults From an Upper-Middle-Income Country: Randomized Controlled Trial. J Med Internet Res 2016 Jan 07;18(1):e5 [FREE Full text] [doi: 10.2196/jmir.5235] [Medline: 26742999]

61. Quinn CC, Shardell MD, Terrin ML, Barr EA, Park D, Shaikh F, et al. Mobile Diabetes Intervention for Glycemic Control in 45- to 64-Year-Old Persons With Type 2 Diabetes. J Appl Gerontol 2016 Feb;35(2):227-243. [doi: 10.1177/0733464814542611] [Medline: 25098253]

62. Royackers A, Regan S, Donelle L. The eShift model of care: informal caregivers' experience of a new model of home-based palliative care. Progress in Palliative Care 2016 Apr 13;24(2):84-92. [doi: 10.1179/1743291x15y.0000000006]

63. Duh ES, Guna J, Pogačnik M, Sodnik J. Applications of Paper and Interactive Prototypes in Designing Telecare Services for Older Adults. J Med Syst 2016 Apr;40(4):92. [doi: 10.1007/s10916-016-0463-z] [Medline: 26860915]

64. Depatie A, Bigbee JL. Rural Older Adult Readiness to Adopt Mobile Health Technology: A Descriptive Study. OJRNHC 2015 May 29;15(1):150-184. [doi: 10.14574/ojrnhc.v15i1.346]

65. Moore AN, Rothpletz AM, Preminger JE. The Effect of Chronological Age on the Acceptance of Internet-Based Hearing Health Care. Am J Audiol 2015 Sep;24(3):280-283. [doi: 10.1044/2015 AJA-14-0082] [Medline: 26649530]

66. Currie M, Philip LJ, Roberts A. Attitudes towards the use and acceptance of eHealth technologies: a case study of older adults living with chronic pain and implications for rural healthcare. BMC Health Serv Res 2015 Apr 16;15:162 [FREE Full text] [doi: 10.1186/s12913-015-0825-0] [Medline: 25888988]

67. Grant LA, Rockwood T, Stennes L. Client Satisfaction with Telehealth in Assisted Living and Homecare. Telemed J E Health 2015 Dec;21(12):987-991. [doi: 10.1089/tmj.2014.0218] [Medline: 26126079]

68. Brenes GA, Danhauer SC, Lyles MF, Hogan PE, Miller ME. Telephone-Delivered Cognitive Behavioral Therapy and Telephone-Delivered Nondirective Supportive Therapy for Rural Older Adults With Generalized Anxiety Disorder: A 
Randomized Clinical Trial. JAMA Psychiatry 2015 Oct;72(10):1012-1020 [FREE Full text] [doi: 10.1001/jamapsychiatry.2015.1154] [Medline: 26244854]

69. Corbett A, Owen A, Hampshire A, Grahn J, Stenton R, Dajani S, et al. The Effect of an Online Cognitive Training Package in Healthy Older Adults: An Online Randomized Controlled Trial. J Am Med Dir Assoc 2015 Nov 01;16(11):990-997. [doi: 10.1016/j.jamda.2015.06.014] [Medline: 26543007]

70. Mavandadi S, Benson A, DiFilippo S, Streim JE, Oslin D. A Telephone-Based Program to Provide Symptom Monitoring Alone vs Symptom Monitoring Plus Care Management for Late-Life Depression and Anxiety: A Randomized Clinical Trial. JAMA Psychiatry 2015 Dec;72(12):1211-1218. [doi: 10.1001/jamapsychiatry.2015.2157] [Medline: 26558530]

71. Egede LE, Acierno R, Knapp RG, Lejuez C, Hernandez-Tejada M, Payne EH, et al. Psychotherapy for depression in older veterans via telemedicine: a randomised, open-label, non-inferiority trial. Lancet Psychiatry 2015 Aug;2(8):693-701. [doi: 10.1016/S2215-0366(15)00122-4] [Medline: 26249300]

72. Chang W, Hou CJ, Wei S, Tsai J, Chen Y, Chen M, et al. Utilization and Clinical Feasibility of a Handheld Remote Electrocardiography Recording Device in Cardiac Arrhythmias and Atrial Fibrillation: A Pilot Study. International Journal of Gerontology 2015 Dec;9(4):206-210. [doi: 10.1016/j.ijge.2015.06.002]

73. Boulos M, Ifeachor E, Zhao P, Escudero J, Carroll C, Costa P, et al. LiveWell-Promoting Healthy Living and Wellbeing for Parkinson Patients through Social Network and ICT Training: Lessons Learnt and Best Practices. International Journal of Healthcare Information Systems and Informatics 2015;10(3):24-41. [doi: 10.4018/IJHISI.2015070102]

74. Diño MJ, de Guzman AB. Using Partial Least Squares (PLS) in Predicting Behavioral Intention for Telehealth Use among Filipino Elderly. Educational Gerontology 2014 Jun 25;41(1):53-68. [doi: 10.1080/03601277.2014.917236]

75. Czaja SJ, Lee CC, Arana N, Nair SN, Sharit J. Use of a telehealth system by older adults with hypertension. J Telemed Telecare 2014 Jun;20(4):184-191 [FREE Full text] [doi: 10.1177/1357633X14533889] [Medline: 24803275]

76. Choi NG, Wilson NL, Sirrianni L, Marinucci ML, Hegel MT. Acceptance of home-based telehealth problem-solving therapy for depressed, low-income homebound older adults: qualitative interviews with the participants and aging-service case managers. Gerontologist 2014 Aug;54(4):704-713 [FREE Full text] [doi: 10.1093/geront/gnt083] [Medline: 23929664]

77. Chi N, Demiris G. A systematic review of telehealth tools and interventions to support family caregivers. J Telemed Telecare 2015 Jan;21(1):37-44 [FREE Full text] [doi: 10.1177/1357633X14562734] [Medline: 25475220]

78. Knowles B, Hanson V. Older Adults' Deployment of 'Distrust'. ACM Trans. Comput.-Hum. Interact 2018 Sep 15;25(4):1-25. [doi: 10.1145/3196490]

\author{
Abbreviations \\ JHNEBP: John Hopkins Nursing Evidence-Based Practice \\ MeSH: Medical Subject Headings \\ mHealth: mobile health \\ RCT: randomized controlled trial \\ WHO: World Health Organization
}

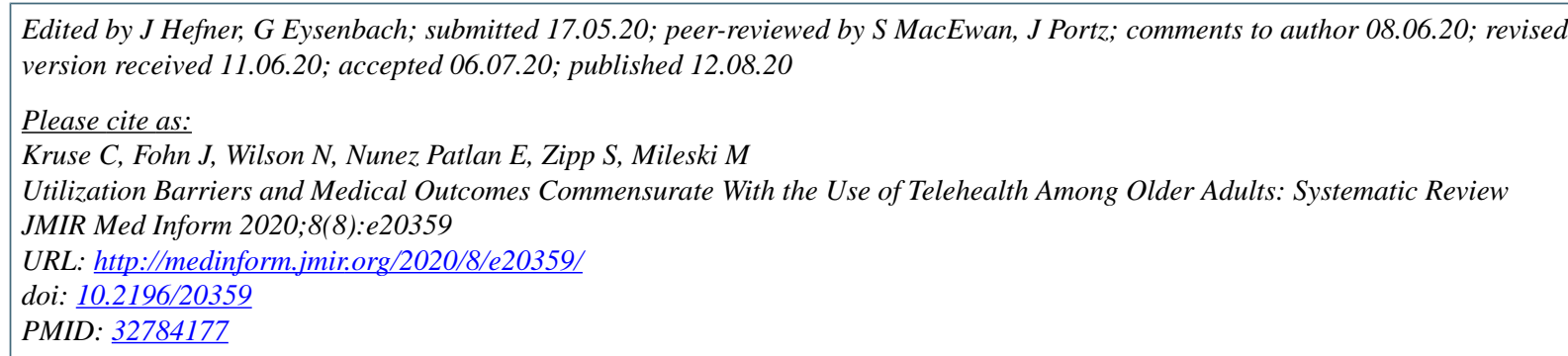

CClemens Kruse, Joanna Fohn, Nakia Wilson, Evangelina Nunez Patlan, Stephanie Zipp, Michael Mileski. Originally published in JMIR Medical Informatics (http://medinform.jmir.org), 12.08.2020. This is an open-access article distributed under the terms of the Creative Commons Attribution License (https://creativecommons.org/licenses/by/4.0/), which permits unrestricted use, distribution, and reproduction in any medium, provided the original work, first published in JMIR Medical Informatics, is properly cited. The complete bibliographic information, a link to the original publication on http://medinform.jmir.org/, as well as this copyright and license information must be included. 